\title{
Flow and Noise Predictions of Coaxial Jets
}

\author{
Anton P. Markesteijn \\ School of Engineering and Material Science, Queen Mary University of London, Mile End Road, \\ London, E1 4NS, UK \\ GPU-prime Ltd, Cambridge, UK
}

Vasily Gryazev

School of Engineering and Material Science, Queen Mary University of London, Mile End Road, London, E1 4NS, UK

Sergey A. Karabasov,

School of Engineering and Material Science, Queen Mary University of London, Mile End Road, London, E1 4NS, UK

GPU-prime Ltd, Cambridge, UK

Lobachevsky State University of Nizhni Novgorod, Nizhni Novgorod, Russia

Ruslan Sh. Ayupov, Leonid A. Benderskiy, Dmitriy A. Lyubimov

Central Institute for Aviation Motor Development named after P. I. Baranov, Russia

Flow and noise solutions of the two Large Eddy Simulation (LES) approaches are evaluated for the jet flow conditions corresponding to a benchmark co-axial jet case from the EU CoJeN (Computation of Coaxial Jet Noise) experiment. The jet is heated and issues for a short-cowl axi-symmetric nozzle with a central body at a transonic speed. The first LES method is based on the Compact Accurately Boundary-Adjusting high-REsolution Technique (CABARET) scheme, for which implementation features include asynchronous time stepping at an optimal Courant-Friedrichs-Lewy (CFL) number, a wall model, and a synthetic turbulence inflow boundary condition. The CABARET LES is implemented on Graphics Processing Units (GPUs). The second LES approach is based on the hybrid 
Reynolds Averaged Navier-Stokes (RANS)/ Implicit LES method that uses a mixture of high-order Roe and WENO scheme and a wall distance model of the Improved Delayed Detached Eddy Simulation (IDDES) type. The RANS/ILES method is run on an MPI cluster. Two grid generation approaches are considered: the unstructured grid using OpenFOAM utility "snappyHexMesh" (sHM) and the conventional structured multiblock body-fitted curvilinear grid. The LES flow solutions are compared with the experiment and also with solutions obtained from the standard axi-symmetric RANS method using the k- $\omega$ turbulence model. For noise predictions, The LES solutions are coupled with the penetrable surface formation of the Ffowcs Williams -Hawkings method. The results of noise predictions are compared with the experiment and the effect of different LES grids and acoustic integration surfaces is discussed.

\section{Introduction}

Development of high-fidelity jet noise prediction models for co-axial jet flows typical of civil turbofan engines to complement and potentially replace the existing scaling laws such as those suggested by [1],[2], and [3] is of great interest to engine manufacturers. Despite this and in comparison with the plenitude of literature on single-stream jet noise [4],[5],[6] the amount of data on dual and multi-stream jet flows is much less. Up to present, only a few experimental campaigns have systematically studied the dual stream jet noise to provide both the flow and the far-field acoustic data for validation of computational methods. One of such databases was generated as the result of the EUfunded Computation of Coaxial Jet Noise project ( CoJeN). The CoJeN jets, whose conditions span across a representative range of jet Mach numbers and core stream temperatures were investigated using, Particle Image Velocimetry (PIV) [7], the near-field acoustic array technique [8], and a combination of PIV and Laser Doppler Anemometry (LDA) [9]. In addition to the experimental investigations, a variety of modeling approaches, ranging from Reynolds Averaged Navier Stokes (RANS) to high-resolution methods such as Large Eddy Simulations (LES) were used for the flow and noise simulation of CoJeN jet flows [10],[11].

The use of LES methods has become increasingly popular for high-fidelity jet noise calculations since, due to their unsteady nature, LES capture a wide diversity of temporal scales typical of high- 
Reynolds number flows [5],[12]. LES are expensive since the resolution of high frequencies typical of noise generation in the initial region of the jet shear layers require very small computational time steps while long simulation times are required to capture a sufficient number of time samples of low frequency noise for achieving statistical stationarity at low frequencies. The cost is especially high for wall-resolved LES methods which remain prohibitively expensive for complex jet flows. A less computationally demanding class of the high-resolution jet noise modeling methods is based on Wall Modeled LES (WMLES) and hybrid RANS-LES schemes [13]. Still, even in the framework of WMLES or Hybrid LES approaches, the cost of solving unsteady Navier-Stokes equations is still typically higher in comparison with simpler methods such as Lattice Boltzmann (LBM) [14]. Furthermore, the LES remain significantly more expensive than RANS routinely used in engineering calculations. Hence, from the practical viewpoint, when the simulation time is an important factor, there is a question what is the "value for money" of LES in comparison with the fast-turn-around-time RANS methods. For example, the low cost of RANS flow solutions methods explain the popularity of low-fidelity methods for coaxial jet flow and noise calculation such as a combination of the RANS solutions and the Lighthill acoustic analogy [15] as well as a semi-analytical investigation of the hydrodynamic stability and noise generation mechanisms in CoJeN jets [16]. Furthermore, RANS solutions of the CoJeN jets were used for noise predictions based on the Goldstein generalized acoustic analogy in [18]. Furthermore, when LES is selected as the method of choice for a complex geometry like the coaxial jet, practical considerations always include questions on the best grid generation strategy and design of appropriate acoustic integration surfaces for noise modelling with the FW-H method.

The goal of the current investigation is to compare computational efficiency of different LESbased approaches for flow and noise modelling for the same well-documented co-axial jet case following the preliminary work presented in [20]. One of the LES methods compared is based on explicit asynchronous time-stepping scheme, which uses a hierarchy of local time steps depending on the local cell size [28]. The Navier-Stokes solver is based on the Compact Accurately BoundaryAdjusting high-REsolution Technique (CABARET) scheme [24],[25],[26],[27],[28],[30] which is implemented with a wall model [31],[32],[33] and a synthetic turbulence boundary condition 
[34],[35]. Because of the computational stencil compactness, CABARET can utilse unstructured grids with patches of refined isotropic Cartesian meshes as required in the vicinity of viscous boundary and jet shear layers [21],[22],[23]. The second LES approach is based on a hybrid RANS-LES method [36],[37] which is of Ideal Delayed Detached Eddy Simulation (IDDES)-type [45]. The method utilises an implicit time-marching scheme, which allows using a large (global) time step and a mixture of the high-order Roe and WENO-type scheme to preserve oscillation-free flow solutions [38]. The method is well-suited for high-quality structured multiblock body-fitted curvilinear meshes with a refinement in the viscous boundary and jet shear layers. In the wall region, which is defined by a wall distance parameter of the RANS-ILES method, RANS equations based on the Spalart-Allmaras turbulence model are solved [39]. Similar to the CABARET LES, there is no explicit SGS (sub-gridscale) turbulence model used in the RANS-LES method so that the role of SGS is played by the scheme viscosity (or flux correction) in accordance with the Implicit LES (ILES) approach. For farfield noise predictions, the LES methods are coupled to the Ffowcs Williams - Hawkings formulation based a permeable control surface with multiple closing disks downstream in the jet flow[19],[12].

In addition to the LES solutions, the standard RANS solution will be considered for the same jet case. The RANS solution is obtained the axisymmetric Navier-Stokes equations with the $\mathrm{k}-\omega$ turbulence model in ANSYS Fluent based on the recommended set of the turbulence model parameters, a body-fitted grid, and a second-order upwind Roe scheme with the standard symmetry and pressure outlet boundary conditions. For subsequent far-field noise predictions, the meanflow velocity, turbulent kinetic energy, and turbulence dissipation rate solutions of the RANS model can be used for predicting the co-axial jet noise, for example, in the framework of the generalized acoustic analogy [17],[18]. Hence, one of the aims of the present work is to evaluate the quality of the RANS flow solution for the same coaxial jet case: how well the key input ingredients of a subsequent acoustic modelling, such as the meanflow velocity and turbulence distribution, can be predicted from RANS in comparison with the higher-fidelity (but more-costly) LES methods.

The selected Operation Point (OP) 1.3 jet case of the CoJeN family corresponds to a well-defined dataset including the short-cowl axi-symmetric nozzle geometry, the flow data downstream of the central body, and the far-field noise measurements. The experiments were conducted in QinetiQ 
Noise Test Facility (NTF) at Farnborough, UK. The inner stream of the considered co-axial jet flow is heated and issues from the core nozzle at supersonic acoustic Mach number while the outer stream is cold and has a subsonic flow velocity. The combination of dual-stream jet parameters leads to transonic flow regions emerging in the jet core/bypass stream shear layers, which makes this benchmark case challenging for modelling.

The structure of the current article, which aims to analyse best practices for coaxial jet flow and noise simulations, is as follows. In Section II, distinct features of the two LES methods are discussed in some details. These include the asynchronous time stepping and the synthetic turbulence inflow boundary condition for the CABARET method and the wall distance model of the suggested hybrid RANS-LES approach. In Section III, conditions of the CoJeN experiment are given including the datum geometry. Two LES grid generation approaches, one is unstructured and the other is structured, are outlined. Both of these approaches will be tested on the CABARET LES solver, which is amenable to unstructured meshes. The same section also contains details of the Ffowcs Williams Hawkings method implementation. Section IV is devoted to validation of the LES flow solutions on different grids in comparison with the experiment, which also includes comparisons with the RANS solutions. In Section V, the acoustic predictions based on the FW-H method coupled with LES are compared with the experiment to probe their sensitivity to the choice of LES grid and acoustic integral surfaces.

\section{Numerical Methods}

The goal of this section is to present characteristic features of the LES methods which have not been considered in detail in previous publications. Both LES methods are implemented for the governing Navier-Stokes equations in dimensionless variables, which allows for their efficient solution in singleprecision arithmetics without any notable loss of accuracy. In both cases, the laminar viscosity and heat diffusion parts of the governing Navier-Stokes equations are approximated with second-order finite-volume schemes. Hence, because of the high Reynolds number jet flow of interest, main attention is given to the approaches used for calculation of the hyperbolic parts including the turbulence modelling and the associated boundary conditions. 


\section{A. CABARET}

To start with the CABARET method, for advection equation that corresponds, the CABARET stencil has one cell in space and time thanks to the use of staggered conservation and flux variables. The two variables are marched in time using the information from the previous time steps with achieving the second-order of accuracy in space and time. The space-time variables are different in comparison with the standard semi-discrete finite-volume schemes, which are based on the computation of conservation fluxes through some interpolation between the conservative states. For approximation of the Euler part of the Navier-Stokes equations, the CABARET method is implemented using the characteristic decomposition approach based on the local Riemann invariants. As a means to provide the implicit sub-grid-scale model in LES, CABARET uses a low-dissipative flux correction based on the maximum principle. Thanks to its computational stencil simplicity and compactness, CABARET is implemented on unstructured hexagonal-dominant meshes of hanging-node type such as snappyHexMesh (sHM) provided by the OpenFOAM grid generator. Furthermore, the current implementation is performed at a single precision arithmetics and with small memory footprint, which makes the CABARET LES feasible for running on Graphics Processing Units (GPUs) thereby making advantage of their compact size, moderate cost, and significant number of computing cores. Three features of the current implementation of CABARET, which make it to stand out in comparison with many other LES methods and also in comparison with the previous CABARET implementations for single-stream jet flow and noise [30] are: (i) asynchronous time stepping, (ii) implemented wall model, and (iii) synthetic turbulence inflow boundary condition.

\section{$\underline{\text { Asynchronous time stepping }}$}

Asynchronous time stepping marches the solution in time with a local time step which is selected so that the target Courant-Friedrichs-Lewy (CFL) number is approximately constant for all grid cells and corresponds to the optimum value for accuracy. For CABARET, the target CFL number corresponds to 0.5 , for which the scheme is exact for one-dimensional linear advection equation. The asynchronous time stepping algorithm is implemented in accordance with the following procedure [28],[29]: 
At each time step, we calculate the minimum and maximum time steps based on the grid resolution and instantaneous flow solutions,

$$
\Delta t^{\min }=0.5\left(h_{q} /\left(u_{q}+c\right)\right)^{\min } \text { and } \Delta t^{\max }=0.5\left(h_{q} /\left(u_{q}+c\right)\right)^{\max }
$$

where $c$ is the local sound speed, $h_{q}$ is the characteristic grid cell size, $u_{q}$ is the local velocity component, and $\mathrm{q}=1,2,3$ are Cartesian coordinates.

Following the dyadic algorithm suggested in [40],[41], we define

$$
2^{M} \leq \frac{\tau_{\max }}{\tau_{\min }}<2^{M+1}
$$

where $M$ is the number of grid cell groups (update groups) which are computed with the same local time step. $M$ depends on the grid refinement as dictated by resolution requirements in the region of large gradients of the flow solution such as near the wall or in the early shear layers.

Next, we allocate each grid cell to one of the update groups, $m=0,1, \ldots, M$, depending the cell size and the local flow velocity, where cells at $m=0$ correspond to the smallest grid region near the wall and $m=M$ is sued for the largest cells away from the turbulent jet flow. Then $\tau_{0}=2^{M} \cdot \tau_{\min }$ is called the synchronisation time step, which defines the time period over which the local times in all grid cells, which have been updated at different rates, are synchronised.

Having started from the synchronized solution at the current time step, the solutions in each cell are updated using their local time steps. Using interpolation between different local times, we march the solutions in cells in time at rates in accordance with their update group, $2^{-m}$ Once the solutions in all cells reach the synchronisation time step $\tau_{0}$, we update the solutions in all cells by redistributing the fluxes between the adjacent cells of different sizes thereby preserving the flux conservation. A useful estimate of the speed-up of the asynchronous time stepping in comparison with the single (global) time stepping algorithm limited by the smallest time step can be obtained from the ratio of the average time step to the smallest time step,

$$
\mathrm{C}_{\mathrm{E}}=\frac{\sum_{\mathrm{i}, \mathrm{q}}\left(h_{q} /\left(u_{q}+c\right)(i)\right) / \mathrm{N}}{\min _{\mathrm{i}, \mathrm{q}}\left(\left(h_{q} /\left(u_{q}+c\right)(i)\right)\right)}
$$


where $1 \leq i \leq N$, and $\mathrm{N}$ is the total number of grid cells.

The use of asynchronous time stepping has a clear advantage for the CoJeN problem. For example, the smallest time step based on the smallest cell in the unstructured sHM mesh results in a time step of $3.4 \times 10-5 D_{j} / U_{j}$ where $D_{j}$ is the bypass nozzle lip diameter and $U_{j}$ is the jet velocity at the core nozzle exit. However, this time step is applied only for a small amount of cells $(\sim 1 \%)$. The number of the update groups of the asynchronous time stepping in this case is 8 , which corresponds to $M=7$. This means that the time step for the largest cells is $2^{M}=128$ times larger $\left(4.9 \times 10-3 D_{j} / U_{j}\right)$. Because of the distribution of the grid cell sizes, the sHM LES simulation has 15 times speed-up compared to the (global) single time stepping based on the smallest time step. On the structured mesh, which was optimized for RANS-LES method near the wall boundary, the size of smallest cell in the wall-normal direction is a factor of 3.75 smaller than that of the sHM grid. This reduces the minimum time step to $9.1 \times 10-6 D_{j} / U_{j}$. However, because the number of the smallest cells, which are clustered in the boundary layer and in the shear layer, is very small, the use of asynchronous time stepping (on the same number of update groups) on the structured grid is even more efficient than that on the sHM grid. Compared to the single time stepping, the speed-up due to the asynchronous time stepping reaches $35 x$. The speedup achieved partly compensates for the decrease of the smallest time step: the CABARET solution on the structured grid is only $40 \%$ slower than that on the unstructured sHM grid.

\section{$\underline{\text { Wall model }}$}

The basic steps of the WMLES algorithm implemented in the CABARET flow solver are as follows. Inside the boundary layer mesh, each time step the cell centered values of the velocity and density are evaluated. These values are provided from CABARET to the wall model, which, in turn, returns the wall shear stress that is used as the wall boundary condition for CABARET. In particular, the wall model that has been employed in the present work is the one based on the algebraic method using Reichardt's law as described in [33]. Reichardt's law of the wall provides a relation between the dimensionless velocity in the boundary layer velocity units, $u^{+}=U / \sqrt{\tau_{w} / \rho}$ and the dimensionless wall coordinate $\mathrm{y}^{+}$. Here $\tau_{w}$ is the shear wall stress, $\rho$ is the local density, $U$ is the local stream-wise 
velocity. $y^{+}=y u^{*} / v$, where $y$ is the wall-normal coordinate of the cell centre nearest to the wall, $u^{*}$ is the friction velocity estimated from the corresponding cell centre velocity, and $v$ is the kinematic viscosity. In accordance with the WMLES framework, the instantaneous velocity $u$ is used as an external boundary condition input for $u+$. The resulting non-linear algebraic equation, using $u, y$, and the laminar viscosity, is solved by a simple Newton iteration, giving the wall shear stress required. Notably, the computational cost of the wall model is negligible (less than $0.1 \%$ ) in comparison with the overall LES cost.

\section{Inflow synthetic turbulence condition at the nozzle inlets}

The turbulent inflow condition in the CABARET method consists of the three components.

For obtaining of random velocity fluctuations in accordance with the synthetic turbulence generation method [34], a periodic box domain is considered. The area of the turbulence box size is adjusted to match the cross-section of the nozzle inlet. The stream-wise dimension of the box is such that it contains 2-3 lengths of $U_{\text {inlet }} \Delta t$, where $U_{\text {inlet }}$ is the stream-wise flow velocity at the nozzle inlet. The value of $U_{\text {inlet }}$ is extrapolated from the interior numerical solution. $\Delta t$ is the local LES time step. Following [42], the von-Karman Pao wavenumber spectrum of the turbulent kinetic energy is used. The number of cells and wave forms in each direction of the box is adjusted so that the generated turbulent structures are resolved within 8-10 points of the LES grid in accordance with its resolution in the wall region. Since the state of the boundary layer at the nozzle exits in the CoJeN experiment is unknown, the magnitude of the input wavenumber spectrum is adjusted so that turbulence intensity at the nozzle exit is about 3\% in agreement with the existing literature on turbulent inflow boundary conditions [43]. The output of the synthetic turbulence generator is random realisations of turbulent velocity fluctuations. The time series of turbulent velocity fluctuations imposed at the nozzle inlet are made consistent with the turbulence convection speed equal to the velocity at the nozzle inlet. This is achieved by first calculating the distance travelled by a turbulent structure $U_{\text {inlet }} t$ for each simulation time moment $t$ and then using the velocity distributions in a cross-stream frame of the turbulence generator box corresponding to this distance for the inflow boundary condition. 
The turbulent velocity fluctuations obtained from the turbulence box generator are superimposed on the meanflow profile of the stream-wise velocity for a channel flow solution, which is obtained from a precursor numerical simulation. The channel cross-section and the stream-wise velocity parameters are matched to those of the nozzle inlet.

The obtained unsteady velocity field at the nozzle cross-section is used in the characteristic boundary conditions of the CABARET scheme applied at the nozzle inlet. The characteristic boundary conditions are based on imposing the local Riemann invariants corresponding to the incoming waves, which for the subsonic inlets of the CoJeN nozzle correspond to one pressure wave, two tangential velocity components, and an entropy wave. The first three invariants include the velocity component specified in accordance with the synthesized incoming turbulent flow solution.

To illustrate the work of the synthetic turbulence boundary condition, Fig.1a shows distributions of the mean stream-wise velocity component and its root-mean-square (r.m.s.) fluctuations inside the CoJeN nozzle. Radial profiles of the mean stream-wise velocity component and its r.m.s. at the bypass and the core nozzle exits are shown in Fig.1b. It can be noted that the distributions of meanflow velocity and turbulent velocity fluctuations, including those inside the core and bypass nozzles, are in a reasonable agreement with the expected shape of a turbulent boundary layer profile.
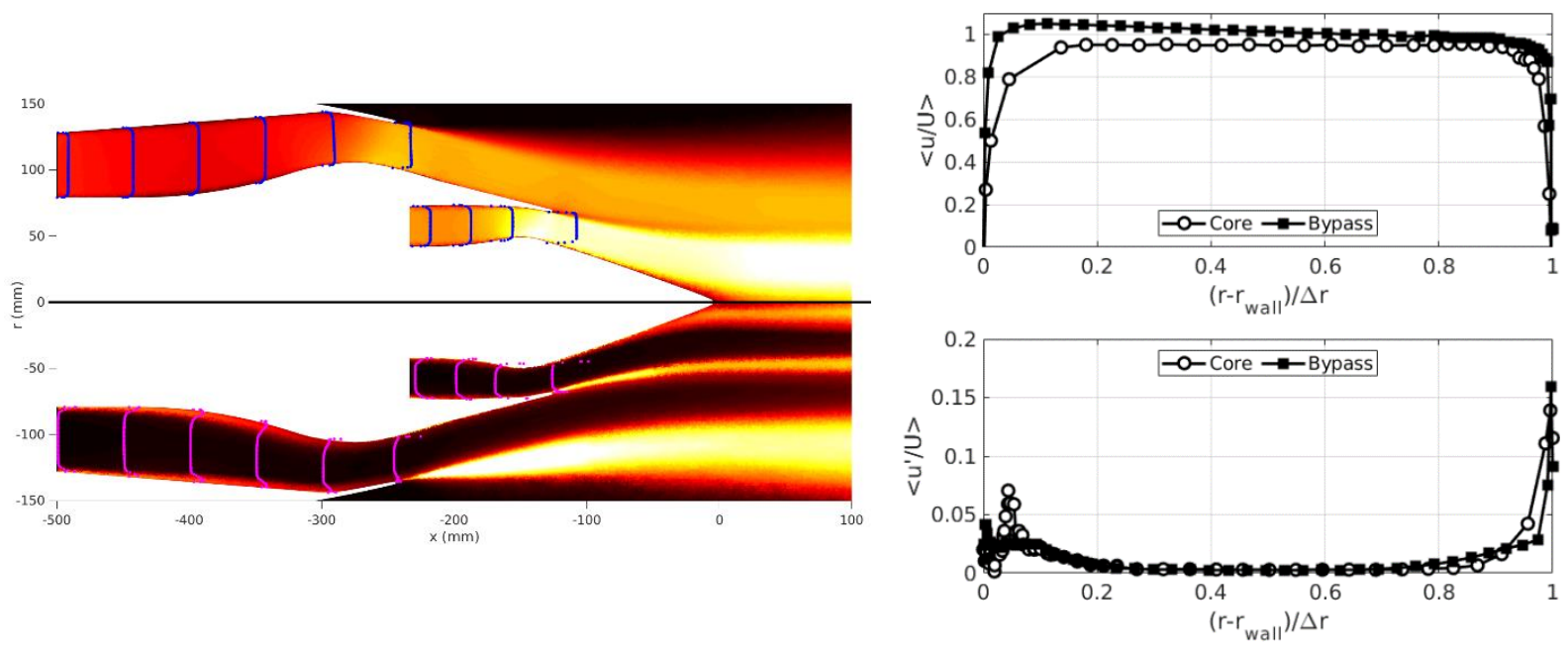

Fig. 1 Effect of the synthetic turbulence boundary condition on meanflow velocity and r.m.s. fluctuations of the stream-wise velocity component: (a) nozzle symmetry plane, (b) radial profiles at the core and bypass nozzle exits. Contours of several velocity and velocity fluctuation 
profiles inside the nozzle streams are shown in (a). The nondimensionalisations used for velocity and radial distance in (b) are based on the core and bypass nozzle parameters.

\section{B. RANS-LES method}

In the RANS/ILES method, standard steady boundary conditions are imposed at the nozzle inlets based on the total pressure and the total temperature of each jet stream. In the solver, the upstream and downstream states of the Roe flux were calculated using a monotone scheme of the $9^{\text {th }}$ order with upwind-biased differences to ensure low numerical dissipation [38]. In the wall region, convective fluxes of the turbulence transport equation were calculated using the same Roe scheme as in the gas dynamics part of the Navier-Stokes equations. The upstream and downstream states were computed using the $5^{\text {th }}$ order WENO method [38] for nozzle inlet and external boundaries. The structured grid is used. For time integration, an implicit time scheme is used with simulation time step $4.8 \times 10-3 D_{j} / U_{j}$.

Similar to other hybrid RANS-LES methods, the same unsteady RANS equations with the SpalartAllmaras turbulence model are solved in both the RANS-region near the wall and the ILES-region by switching conditions between RANS and ILES. The switching is based on the modified wall distance $d_{I L E S}$ to nullify turbulent viscosity in ILES-region. A wall function or no-slip condition is defined depending on the wall-normal coordinate $y^{+}$of the center of the grid cell next to the wall. The modification is achieved by adjusting the distance in the dissipative term in the equation of the turbulence model, which procedure details are available in [36],[37], and [39]. Notably, the modified distance definition is slightly different from the conventional Detached Eddy Simulation (DES) methods and calculated using the equations as follows:

$$
\begin{aligned}
& d_{I L E S}=\max \left(d_{\Delta}, d_{f}\right) \\
& d_{\Delta}=d, \text { under } d \leq C_{I L E S} \times \Delta_{\text {max }} ; d_{\Delta}=10^{-6} H, \text { under } d>C_{I L E S} \times \Delta_{\text {max }} \\
& d_{f}=d \times f_{d}, \text { under } f_{d} \geq f_{d \min } ; d_{f}=10^{-6} H, \text { under } f_{d}<f_{d \min } \\
& f_{d}=\max \left(f_{d t}, f_{b}\right)\left(1+f_{e} \Psi\right) \\
& f_{d t}=\tanh \left(\left(C_{d t} r_{d t}\right)^{3}\right)
\end{aligned}
$$


Here $d$ is the distance from the wall to the center of the current cell, $C_{I L E S}=1, f_{d \min }=0.01, H$ is characteristic length, and $\Delta_{\max }$ is the maximum size of this grid cell. It should be noted that the role of $C_{I L E S}$ differs from $C_{D E S}$ in the DES method [44]. Indeed, the parameter $C_{D E S}$ in DES has two functions: it determines the position of the transition from RANS to LES and, more importantly, is explicitly included in the expression of the Sub Grid Scale (SGS) turbulence model so that the value of the sub-grid viscosity depends on this parameter. Such explicit dependency would not be ideal for relatively dissipative schemes such as those based on upwind finite-differences for convective fluxes as used in the RANS-ILES method. This is because the effective turbulent viscosity of the method is a sum of the sub-grid-scale model viscosity and the viscosity of the numerical scheme. Therefore, in the case of the RANS/ILES method, the $C_{\text {ILES }}$ parameter only affects the location of the transition from RANS to ILES but not the actual SGS viscosity value. For this reason, the effect of the numerical dissipation on the solution is reduced. It can be further noted that equation (7), which defines $f_{d}$ has a similar functional dependency to the one used in Improved Delayed Detached Eddy Simulations (IDDES) [45]. Function $f_{d t}$ is defined by (8), where $C_{d t}=8$. Other functions in the above equations are the same as those provided in [45].

\section{Nozzle geometry, computational grids, acoustic surfaces, and simulation run times}

\section{A. SCN nozzle}

The considered nozzle geometry corresponds to the axisymmetric co-axial short-cowl nozzle (SCN), which detailed description can be found in [8]. The total temperature ratios, $\frac{T_{o b}}{T_{o j}}=0.38$ and bypass ratio $\frac{U_{b}}{U_{j}}=0.64$ were specified according to the conditions shown in table 1 , where the subscripts $j$ and $b$ denote core (primary) and bypass (secondary) nozzles/jets, respectively. The sound speed, which was used to calculate the jet Mach numbers in table 1, was computed based on the corresponding flow temperature (primary or secondary) using $c=\sqrt{\gamma R T_{s j}}$ or $\sqrt{\gamma R T_{s b}}$, where $T_{s j}$ and $T_{s b}$ correspond to the local static temperatures in the core and bypass jet streams, and values for the gas constant, $R$ and the ratio of specific heats, $\gamma$ of $287 \frac{\mathrm{J}}{\mathrm{kg} \cdot \mathrm{K}}$ and 1.4, respectively. The sound speed 
for the ambient field at normal temperature and pressure conditions, $T_{\infty}=288.14 \circ \mathrm{K}$ and $P_{\infty}=99703$ $\mathrm{Pa}$, is $341 \mathrm{~m} / \mathrm{s}$.

A detailed drawing, showing all relevant dimensions of the SCN geometry, can be found in [8], Fig.3. In according with the drawing, the values for the lip diameters of the core and bypass nozzles are $0.136 \mathrm{~m}$ and $0.273 \mathrm{~m}$, respectively, the lip angle tangents of the core and bypass nozzles are 0.275 and 0.191 , respectively, and the bullet angle tangent is 0.363 .

The bypass nozzle lip diameter, $D_{j}(0.273 \mathrm{~m})$ and the core jet stream velocity, $U_{j}(480.7 \mathrm{~m} / \mathrm{s})$ are further used as reference length and velocity scales in the rest of the article. The Reynolds number of the jet based on these two parameters is $3.010^{6}$.

Table 1. Main parameters of the OP1.3 CoJeN case

\begin{tabular}{|c|c|c|c|}
\hline \multicolumn{2}{|c|}{ Core } & \multicolumn{2}{c|}{ Bypass $\left(\mathrm{D}_{\mathrm{j}}=\mathbf{0 . 2 7 3 m}\right)$} \\
\hline $\mathrm{U}_{\mathrm{j}}(\mathrm{m} / \mathrm{s})$ & 480.7 & $\mathrm{U}_{\mathrm{b}}(\mathrm{m} / \mathrm{s})$ & 306.8 \\
\hline $\mathrm{M}_{\mathrm{j}}$ & 0.877 & $\mathrm{M}_{\mathrm{b}}$ & 0.902 \\
\hline $\mathrm{T}_{\mathrm{sj}}(\mathrm{K})$ & 775.6 & $\mathrm{~T}_{\mathrm{sb}}(\mathrm{K})$ & 288.14 \\
\hline $\mathrm{T}_{\mathrm{tj}}(\mathrm{K})$ & 827.9 & $\mathrm{~T}_{\mathrm{tb}}(\mathrm{K})$ & 335.0 \\
\hline
\end{tabular}

\section{B. Computational Grids}

\section{$\underline{\text { Unstructured snappyHexMesh }}$}

The first grid generation approach considered corresponds to the semi-automatic mesh generation process using the OpenFOAM utility "snappyHexMesh" (sHM). This mesh utility enables hexadominant mesh generation from triangulated surface geometries (e.g. CAD geometry), an essential need for the LES treatment of complex geometries. Importantly, the mesh generation utility allows to generate locally fine patches of good quality Cartesian grid in the jet shear layers, where the azimuthal grid resolution is the standard bottle-neck of structured multiblock curvilinear grids. Furthermore, the sHM utility also has the capability to "snap" the mesh to the geometry as well as the ability to generate "layers" of body-fitted grid near the boundaries. The stream-wise dimension of the computational domain is about $100 D_{j}$ and the other two dimensions of the mesh are $30 D_{j}$ each. Fig.2 
shows mesh elements in the 3D volume around the CoJeN nozzle geometry including zoom-in views in the symmetry plane of the nozzle. Importantly, the size and thickness of the grid layers of sHM near the boundary can be precisely controlled, which involves adding body-fitted hexahedral layers near the viscous wall boundary. During the automatic meshing procedure, the distance between the centre of the control volume closest to the boundary and the boundary itself is kept within a prescribed distance. Furthermore, a good quality Cartesian mesh is obtained in the region of the jet shear layers with taking into account the jet spreading. The unstructured LES grid generated by this method has $82 \times 10^{6}$ cells, which include a 3 cells per target boundary layer thickness $\left(0.0018 D_{j}\right.$ inside the nozzle) with an expansion ratio of 2.0. In terms of the wall-normal grid size units $y^{+}$, the grid resolution at the nozzle exit is estimated to be $20-30$ based on the Reynolds number of the flow in the experiment. The nozzle lip cell sizes in the axial and radial directions are $0.0011 D_{j}$ and $0.00026 D_{j}$. Specific attention is given to the initial regions shear layer development, where extra refinement regions are placed after each nozzle lip. Thanks to the refinement, the number of grid cells in the initial shear layers around the jet circumference is kept sufficiently high: around 800 and 1400 for the core and bypass nozzle, respectively. Furthermore, because of the expected wake after the central body, a patch of dense Cartesian mesh is used is placed downstream of the bullet body. In each case, the grid refinement locations are determined from an initial low-resolution run of the same mesh and flow conditions. The final refined mesh is generated by the sHM routine using a script and takes several hours on $16 \mathrm{CPU}$ cores without further user interaction.

It can be noted that one downside of the sHM grid generation approach which is the lack of smoothness in the areas of local grid refinement. For this reason, and also due its unstructured hanging-node nature, the sHM has only been used in the CABARET LES calculations. Importantly, thanks to the compactness of the CABARET stencil, the extension of its basic algorithm to nonuniform meshes of the sHM type is performed without a notable effect on the solution quality. 


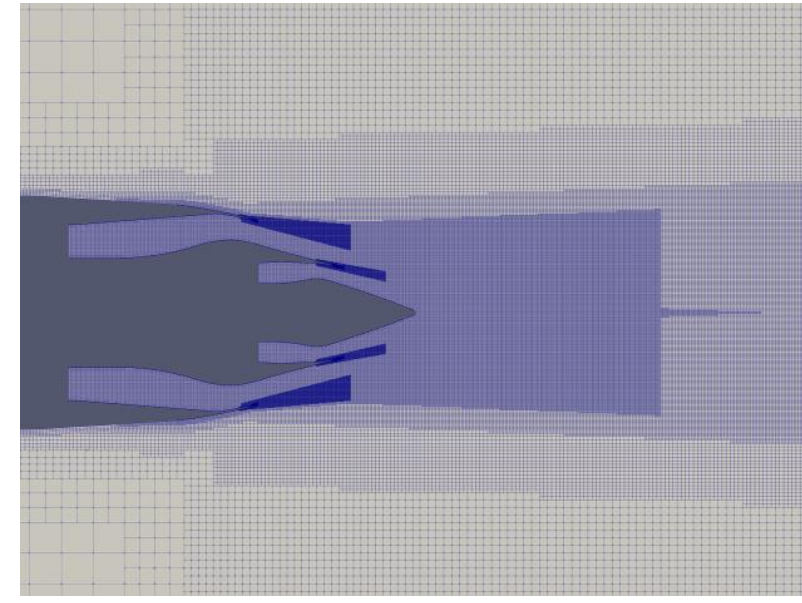

(a)

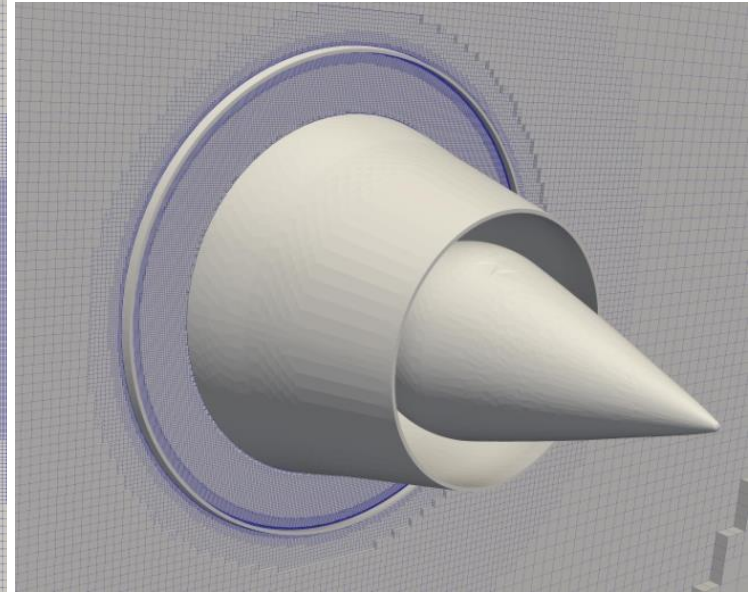

(b)

Fig. 2 Unstructured LES grid (sHM): a cross section in the symmetry plane (a) and grid details in a cross-stream plane (b).

\section{$\underline{\text { Structured Body-Fitted Grid }}$}

A structured multiblock body-fitted grid for the CoJeN problem is created using ESI CFD-GEOM mesh generator based on the previous experience in turbofan engine nozzle simulations. In the process of the final mesh generation, several iterations are performed to optimize the direction of the fine mesh cones from the nozzle lips in order to obtain a sufficient resolution of mixing layer eddies. The final structured mesh consists of $78 \times 10^{6}$ control volumes (Fig. 3). The entire length of the computational domain is about $30 D_{j}$ and the diameter of the mesh cone grows from about $15 D_{j}$ to $24 D_{j}$. The number of cells in the jet circumferential direction is 240 . For the bypass nozzle lip, the cell size in the boundary layer and the initial shear layer in the axial and the radial direction are about $0.00073 D_{j}$ and $0.00026 D_{j}$, respectively. The resolution for the same at the core nozzle lip is $0.00069 D_{j}$ and $0.00020 D_{j}$.

In comparison with the unstructured sHM mesh, the structured mesh provides a factor of 3.75 resolution in the wall-normal direction and is smoother. In fact, the mesh element distribution in the axisymmetric plane of the structured LES grid is much closer to the body-fitted curvilinear grid (Fig.4) for 3D-axisymmetric RANS calculations than to the unstructured sHM grid. Notably, the RANS grid has similar axial and radial dimensions in comparison with the structured LES grid and 
was generated using a similar meshing platform. On the other hand, thanks to the local Cartesian grid refinement, the sHM grid is much more uniform in the shear layer regions, e.g. it has 4-6 times more grid resolution in the circumferential jet direction in comparison with the structured grid. Furthermore, the sHM grid also maintains a good Cartesian quality near the complex nozzle geometry elements such as the nozzle bullet, where preserving the same grid properties using a structured nonuniform grid approach could be a challenge.

The generated structured multiblock grid has been used in both the RANS/ILES method, which requires a good-quality structured block hexahedral mesh and the CABARET method, which is less demanding in terms of the grid smoothness.

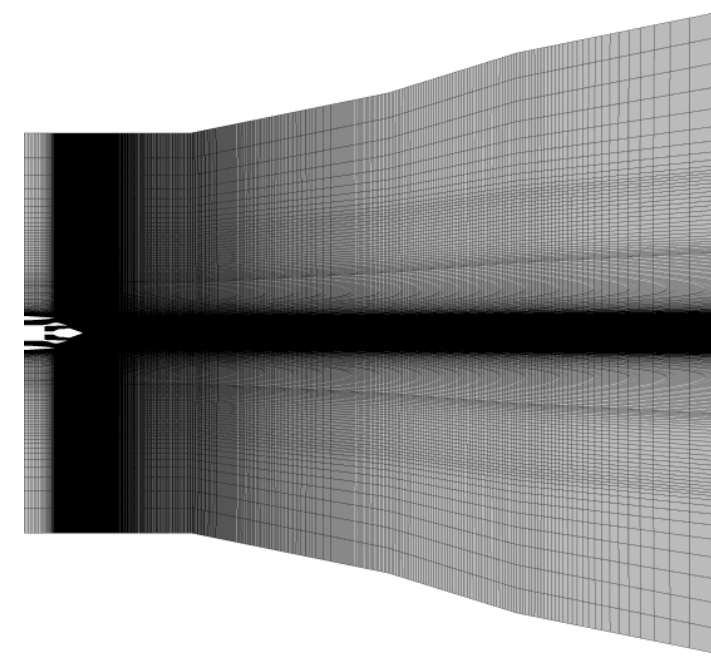

(a)

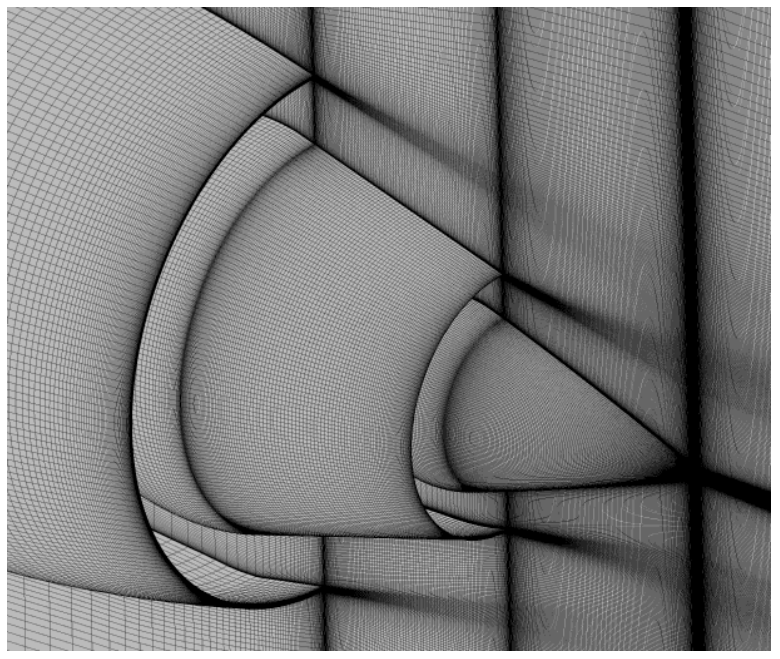

(b)

Fig. 3. Structured multiblock body-fitted LES grid: a cross-section of the full computational domain in the symmetry plane (a) and a cross-section view near the nozzle (b). 


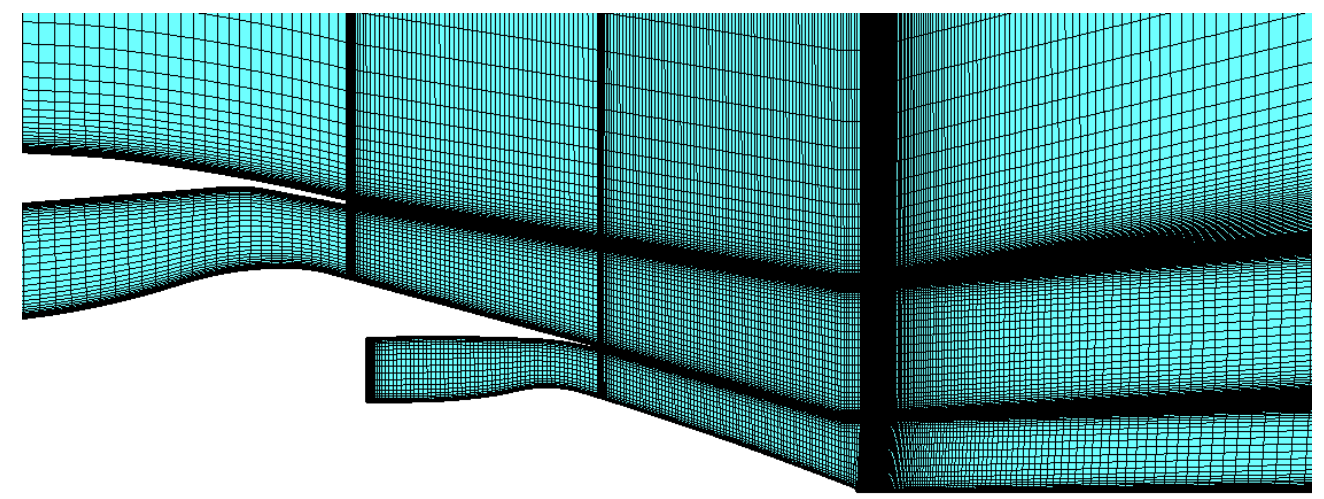

Fig. 4 A multiblock body-fitted curvilinear mesh used in 3D-axisymmytic RANS calculations: the total grid count is $250 \times 10^{3}$ elements and the target value of $y^{+}$is 1 .

\section{Ffowcs Williams -Hawkings integration surfaces}

For the far-field noise calculations, the standard retarded-time time-domain Ffowcs Williams Hawkings (FW-H) method is used with permeable acoustic integration surfaces and multiple closing discs [12],[19]. In the FW-H method, the LES solution is recorded on a set of integration surfaces, which confine the jet turbulence and main vorticity regions in the jet shear layers, and then integrated with the analytical free-space Green's function solution to project the LES solution to the far-field sound.

The acoustic spectra are computed for several polar angles to the jet flow corresponding to a geometrical far-field location at $73 D_{j}$ from the trailing edge of the central body of the nozzle. For converting the time signal obtained from the FW-H calculation to the far-field pressure power spectral density based on the common definition of $1 \mathrm{~Hz}$ as the reference frequency and $20 \mu \mathrm{Pa}$ as the reference pressure, the standard statistical post-processing is applied based on the Welch method to deal with the Fourier transform of short time signal series. In the framework of the Welch method, the original LES signal is broken down into several intervals with 50\% overlap, each of the intervals is Fourier transformed, and the final result is obtained by ensemble averaging over the intervals. Further details of the LES signal post-processing method can be found in [46].

Using points of the LES grid, acoustic integration surfaces are constructed in such a way to contain all significant noise sources. Following recommendations from the literature [12], the acoustic 
surfaces are of a funnel shape to remain parallel to the developing shear layers. At the outlet, the surfaces are terminated with several closing discs designed to avoid the pseudo-sound artifacts by averaging the time signals produced by each individual disc. The position of the acoustic control surface around the jet and its length including the closing discs are adjusted depending on the LES grid in order to ensure a sufficient resolution of acoustic waves.

For the unstructured sHM grid (Fig.5), the position of the conical FW-H surface was placed so that it starts one diameter upstream from the core nozzle lip and extends for about $22 D_{j}$ in the axial direction, while the conical surface follows the jet expansion. The region from $18 D_{j}$ to $22 D_{j}$ is used for 16 closing-discs based on previous experience with this grid. The grid resolution corresponding to the acoustic surface location allows resolving acoustic waves at 8 grid points per wavelength for frequencies from $S_{D}=20$ in the vicinity of the early shear layers to $S_{D}=3$ at the furthest closing disc location. Here the Strouhal number is based on the bypass nozzle exit parameters, $S_{D}=f D_{j} / U_{b}$ where $f$ is dimensional sound frequency. As noted earlier, in this work, the sHM grid and the associated acoustic surfaces have been used with CABARET only.

The structured multiblock grid is shorter in the axial direction in comparison with the sHM grid. Hence, for this grid, a shorter FW-H surface is selected $\left(\sim 17 D_{j}\right)$ in comparison with the sHM acoustic surface. To test sensitivity of the far-field noise predictions, three slightly different control surfaces are considered: (i) the acoustic surface of a similar conical shape and size of the closing-disc zone in comparison with the control surface on the sHM grid (Fig.6, compare with Fig.5) and (ii) two further acoustic surfaces, one of which has a slightly wider cone angle (shown in Fig.7) in comparison with (i) and the other one that has the same cone angle but starts further upstream from the bypass nozzle lip. Acoustic surfaces (ii) were constructed based on the previous experience with the RANS-ILES code [47],[48]. The surfaces are closed with 3 discs with a larger space in-between in comparison with acoustic surface (i), which contains 16 closing discs. Furthermore, to investigate sensitivity of the far-field acoustic solutions to the number of closing discs, further variations of acoustic surface (i) are considered with a smaller number of the closing discs: 3 and 2. The configurations of surface (i) with 2 and 3 closing discs are most similar to acoustic surfaces (ii). The highest resolved Strouhal 
number at the control surfaces of the structured grid at 8 points per wavelength corresponds to $S_{D}=$ $10-20$ in the beginning of the control surface and $S_{D}=1$ at the furthest closing disc.

For far-field sound predictions, the RANS-ILES solution is used in combination with acoustic surfaces (ii). And CABARET LES is used for obtaining noise predictions for acoustic surfaces (i). For consistency, in each case, the far-field noise calculations are conducted in double precision.

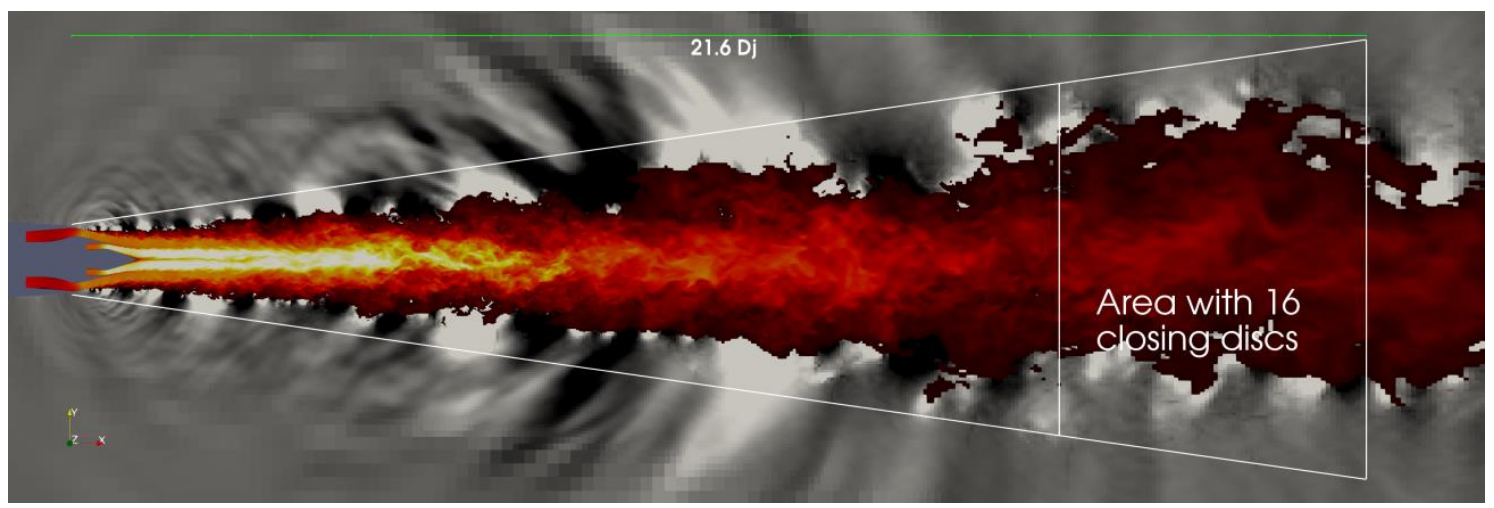

Fig. 5 Acoustic surfaces on the unstructured SHM grid: instantaneous jet velocity and pressure fields (axial velocity magnitude $(\mathrm{m} / \mathrm{s})[0,480]$, pressure amplitudes, $\left.P-P_{\infty} \quad(\mathrm{Pa})[-300,300]\right)$ of the CABARET LES solution and locations of the acoustic integration surface with a region of the closing discs.

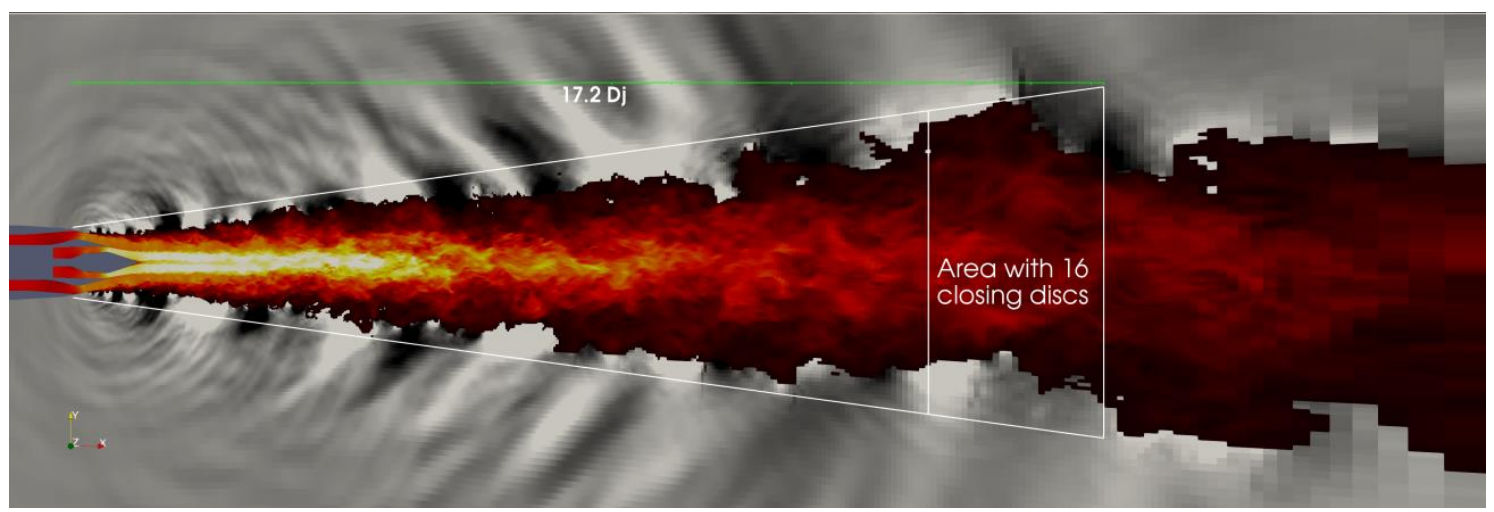

Fig. 6 Acoustic surfaces (i) on the structured multiblock body-fitted grid: instantaneous jet velocity and pressure fields (axial velocity magnitude $(\mathrm{m} / \mathrm{s})[0,480]$, pressure amplitudes, $P-P_{\infty}$ 
(Pa) $[-300,300])$ of the CABARET LES solution and locations of the acoustic integration surface with a region of the closing discs.

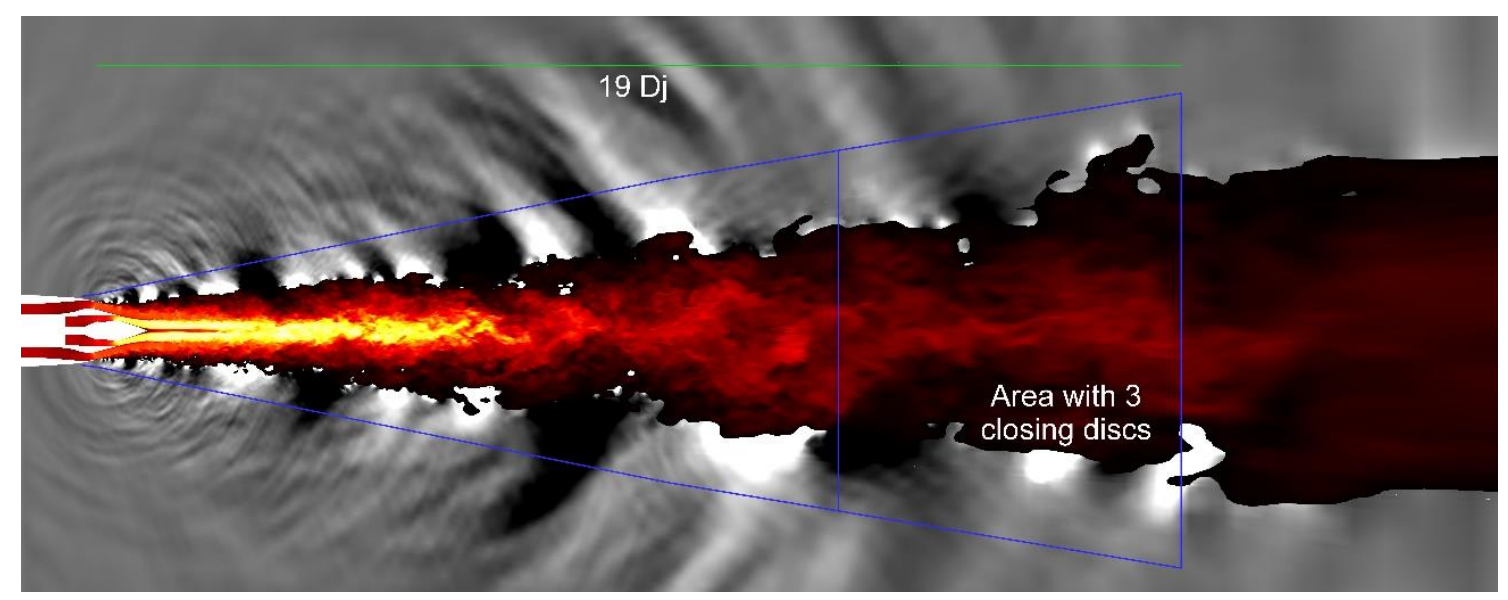

Fig. 7 Acoustic surfaces (ii) on the structured multiblock body-fitted grid: instantaneous jet velocity and pressure fields (axial velocity magnitude $(\mathrm{m} / \mathbf{s})[0,480]$, pressure amplitudes, $P-P_{\infty}$ (Pa) $[-300,300])$ of the RANS/ILES solution and locations of the two acoustic integration surfaces with a region of the closing discs.

\section{LES run times}

Long simulation run times or substantial computational requirements are well-known drawback of LES methods in comparison with RANS simulations, which for the CoJeN problem takes about one hour on a laptop. Hence, computational requirements of the LES methods on different grids are considered in detail here.

The CABARET LES calculations are performed on a workstation equipped with 2 Titan RTX (24GB) GPU cards. 200 TUs (convective time units, $1 \mathrm{TU}=D_{j} / U_{j}$ ) are used as the spin-out time to reach a statistically stationary LES solution and the solution collection time for the subsequent statistical analysis corresponds to a further 400 TUs. The total time to the solution of the GPUCABARET method is 600TUs. The RANS/ILES solutions obtained on an MPI computer cluster of 50 nodes with 2 Intel Xeon E5-2697a_v4 processors per node which amounts to 1600 computer cores altogether. 480 TUs are used to achieve a statistically stationary flow solution and the data are 
collected for a further 340 TUs for statistical processing. The total time to the solution of the RANS/ILES method is 820TUs.

It can be noted that 300 TUs are sufficient for statistical convergence of the LES solutions including the low frequencies important for jet noise, hence both the CABARET LES and the RANSILES solutions are sufficiently well converged. The difference between the LES spin-out times is attributed to different treatments of the initial flow solution transients in the two LES methods which are based on very different time-stepping approaches.

LES run times on the sHM grid and the structured multiblock grid are summarised in Table 2.

Table 2. Amount of TUs obtained from 24 hours of LES run

\begin{tabular}{|c|c|c|c|}
\hline \multicolumn{2}{|c|}{ Unstructured sHM grid } & \multicolumn{2}{|c|}{ Structured multiblock grid } \\
\hline $\begin{array}{l}\text { CABARET on } 2 \mathrm{x} \\
\text { NVidia Titan RTX } \\
(24 \mathrm{~GB})\end{array}$ & $\begin{array}{l}\text { CABARET on } 4 \mathrm{x} \\
\text { NVidia GTX } 1070 \\
(8 \mathrm{~GB}) \\
(24 \mathrm{~GB})\end{array}$ & $\begin{array}{l}\text { CABARET on } 2 \mathrm{x} \\
\text { NVidia Titan RTX } \\
(24 \mathrm{~GB})\end{array}$ & $\begin{array}{l}\text { RANS/ILES on } \\
50 \text { x Intel Xeon E5- } \\
\text { 2697a_v4 }\end{array}$ \\
\hline 100 & 60 & 60 & 55 \\
\hline
\end{tabular}

It is further interesting to analyse the acceleration of the LES solution by reducing the grid size while using the same number of computing cores and the same computer architecture. Such analysis can be straightforwardly performed with the CABARET LES thanks to its use of unstructured grids. Two supplementary unstructured sHM grids are generated with the total grid count of about $12 \times 10^{6}$ cells each. The first grid corresponds to a factor of 2 coarsening in each direction in comparison with the baseline $82 \times 10^{6}$ cell sHM grid. The second grid corresponds to keeping the smallest cells in the boundary layer of the same size as in the $82 \times 10^{6}$ cell grid and coarsening the grid density outside of the boundary layer by a factor of 2 in each direction. The resulting CABARET LES run times on the considered three sHM grids are compared in Table 3. In all three cases, the same number of update groups of the asynchronous time stepping algorithm are used $(M=7)$. It can be noted that the 
CABARET shows a factor of 8.5 acceleration for the uniformly coarsened LES grid case. In comparison with this, for the grid coarsening case with retaining the smallest grid cells in the boundary layer, the achieved acceleration is less, x6.7 due to a reduction of the minimum local time step. Notably, the asynchronous time stepping algorithms helps maintaining a good scalability of LES calculations on both these grids despite a factor of 2 difference in the smallest grid size between the cases.

Table 3. Amount of TUs obtained from 24 hours of CABARET LES on 2x NVidia Titan RTX2 (24 GB)

\begin{tabular}{|c|c|c|}
\hline $\begin{array}{l}\text { Baseline sHM grid of } 82 \times 10^{6} \\
\text { cell }\end{array}$ & $\begin{array}{l}\text { Supplementary } \mathrm{sHM} \text { grid of } \\
12 \times 10^{6} \text { cells with } 2 \mathrm{x} \\
\text { coarsening in all } 3 \text { directions }\end{array}$ & $\begin{array}{l}\text { Supplementary sHM grid of } \\
12 \times 10^{6} \text { cells with } 2 \mathrm{x} \\
\text { coarsening in all } 3 \text { directions } \\
\text { but keeping the smallest cells } \\
\text { in the boundary layer }\end{array}$ \\
\hline 100 & 850 & 670 \\
\hline
\end{tabular}

\section{Results}

\section{A. Flow solutions}

Following [14], results of the LES solutions for several radial profiles of the axial meanflow velocity component and time-averaged turbulent kinetic energy are compared with the experiment (digitized data from [14]) in Fig.8. Fig.8a compares the flow solutions of the CABARET LES method on the baseline sHM grid and those of the RANS/ILES method on the structured multiblock grid with the experiment. The RANS solutions are included in the same figure for reference. To further analyse the flow solution dependence on LES grid while keeping all other parameters the same, Fig.8b compares CABARET solutions on the sHM and structured multiblock grids with the experiment. Furthermore, Fig.9 compares the centerline distributions for the same methods. For example, Fig.9a shows the centerline meanflow velocity distributions for all four solutions compared with the experiment (CABARET on sHM, CABARET on the structured grid, RANS/ILES on the structured 
grid, and RANS). Fig.9b show the root-mean-square (r.m.s.) fluctuations of the stream-wise velocity component for the same four solutions. The experimental data for r.m.s. velocity fluctuations are obtained by digitizing from [11]. To compare with the available centreline distributions from the experiment, the RANS solution for r.ms. of the stream-wise velocity fluctuation is computed from the turbulent kinetic energy following a commonly used relation between the turbulent velocity fluctuation in the stream-wise direction $\left\langle u_{1}^{\prime}\right\rangle$ and other two directions of the high-speed axisymmetric jet flow $\left\langle u_{1}^{\prime}\right\rangle=1.5\left\langle u_{2}^{\prime}\right\rangle=1.5\left\langle u_{3}^{\prime}\right\rangle$ (where 1 is in the jet flow direction and 2 and 3 are in the transverse plane normal to the jet).

The mean axial velocity distributions of the CABARET on the sHM grid and the RANS/ILES on the structured almost coincide one with another. The only notable difference occurs for jet locations close to the centerline for $x / D_{j} \leq 1.65$ where the RANS/ILES solution over-emphasises the velocity deficit in the wake behind the nozzle bullet. The CABARET solution for the steam-wise velocity distribution on the structured grid is overamplified downstream of the central body along the jet centerline and but remains closer to the experiment downstream in the jet (compare Figs.8a, 8b and 9a). The differences between the CABARET and RANS/ILES solutions in the initial jet region is likely to be caused by the effect of the synthetic turbulent inflow condition of the CABARET LES as well as by the non-uniform high-aspect ratio cells of the structured multiblock grid downstream of the nozzle bullet.

Both the CABARET and the RANS/ILES show some amplification of the peak velocity amplitudes in the jet streams starting from the vicinity of the nozzle exit (Fig.8a). This is likely due to the fact that the boundary condition of the experiment at the nozzle exit was not reproduced precisely in either of the LES solutions. The peak velocity amplification in the LES becomes slightly more notable in the fully mixed coaxial jet region at $x / D_{j} \geq 4.21$ where both the LES grids become coarser in comparison with the initial jet flow region thereby reducing the accuracy of modelling the smallscale turbulent mixing. This is especially the case for the sHM grid, which has a good resolution upto $x / D_{j}=4-5$ and then becomes coarser than the structured multi-bock grid. This is demonstrated in Fig.8b by comparing the CABARET solutions for the meanflow velocity on the two grids. 
The comparison of the LES solutions for turbulent kinetic energy follows a similar trend to the meanflow velocity solutions. The LES solutions on the two grids are close one to another and show somewhat amplified peak levels in comparison with the experiment. The RANS/ILES solution on the structured grid overemphasises the deep of turbulent kinetic energy which develops along the jet centerline at region at $x / D_{j} \sim 4$ while the CABARET solution on the sHM grid overpredicts the turbulent kinetic energy at larger distances $x / D_{j}>5$ (Fig.8a). Again, both features can be explained by differences in the grid resolutions: the sHM grid is more uniform and higher quality closer to the nozzle whereas the structured grid maintains a better resolution over a longer distance downstream in the jet. For larger distances downstream in the jet, both LES solutions in Fig.8a show some amplification of the peaks of the turbulent kinetic energy distribution. The latter is consistent with the lack of jet mixing already revealed by inspection of the mean velocity profiles at these distances.

Due to high-aspect-ratio anisotropic cells of the structured grid downstream of the nozzle bullet, the asynchronous time stepping procedure of the CABARET method cannot preserve the same local CFL number optimal for accuracy in all grid directions. As the result, the CABARET solution on the structured grid tends to suppress turbulent velocity fluctuations in the initial jet region due to a possible flow "relaminarisation" and later downstream in the jet, at $x / D_{j}=2.38$ it transitions to turbulence again with generating overamplified peaks of the turbulent kinetic energy in jet shear layers (compare Fig.8b and Fig.9b). Importantly, from comparison of the two CABARET LES solutions with the same inflow boundary condition, the effect of anisotropic grid cells in the initial jet flow region appears to be more dominant in comparison with the effect of the synthetic turbulent inflow boundary condition. Further downstream in the jet, the quality of the structured grid improves especially in comparison with the sHM grid for $x / D_{j}>5$, which leads to an improved agreement of the experiment in these jet regions.

Overall, it can be seen that the agreement between the LES solutions and the experiment is generally good for jet locations $0.92 \leq x / D_{j} \leq 4.21$. This range of jet locations covers the second part of the initial region, the interaction region, and the beginning of the mixed flow region, which contain dominant noise sources of the co-axial jet. The solutions on the sHM grid tend to be more 
accurate in the initial jet flow region. The solutions on the structured multiblock grid tend to more accurate in the downstream part of the jet.

In comparison with the LES solutions, the RANS solution shows a bigger variation with the experiment. In particular, the RANS solution for the meanflow velocity is in a good agreement with LES for the radial profiles (Fig.8a). For the centerline velocity distribution, the RANS meanflow velocity is offset corresponding to some $20 \%$ overprediction of the jet potential core length in comparison with the LES (Fig.9a). The turbulent kinetic energy from RANS shows more differences from the LES and the experiment especially along the jet centerline location, where the RANS solution notably amplifies the turbulent kinetic energy deficit in the coaxial jet interaction region, $2.38 \leq x / D_{j} \leq 4.21$. Furthermore, the RANS solution underpredicts the decay rate of turbulent kinetic energy further outside from the jet edge in comparison with the LES solution. And, due to the overpredicted potential core length, the centerline profile of turbulent velocity fluctuations of the RANS solution is also offset in comparison with the LES and the experiment (Fig.9b). All these discrepancies are due to the lack of the large-scale mixing in the RANS model. Still, interestingly, the RANS predictions of the peak turbulent kinetic energy in the bypass/ambient shear layers, which are most important for low-order jet noise modelling [18], are in a reasonable agreement with LES for a good range of jet locations within $0.55 \leq \frac{x}{D_{j}} \leq 6.41$ (Fig.8a).

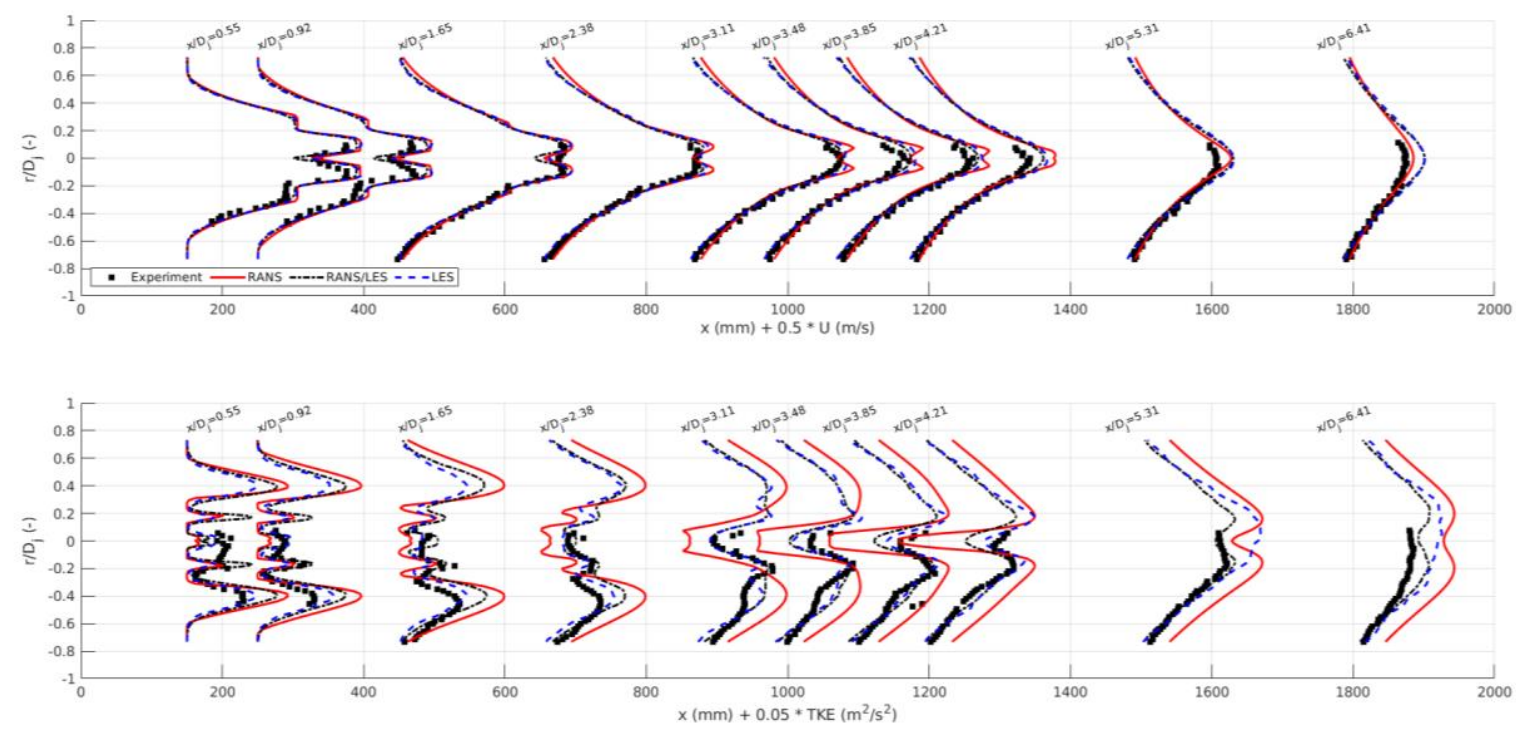

(a) 

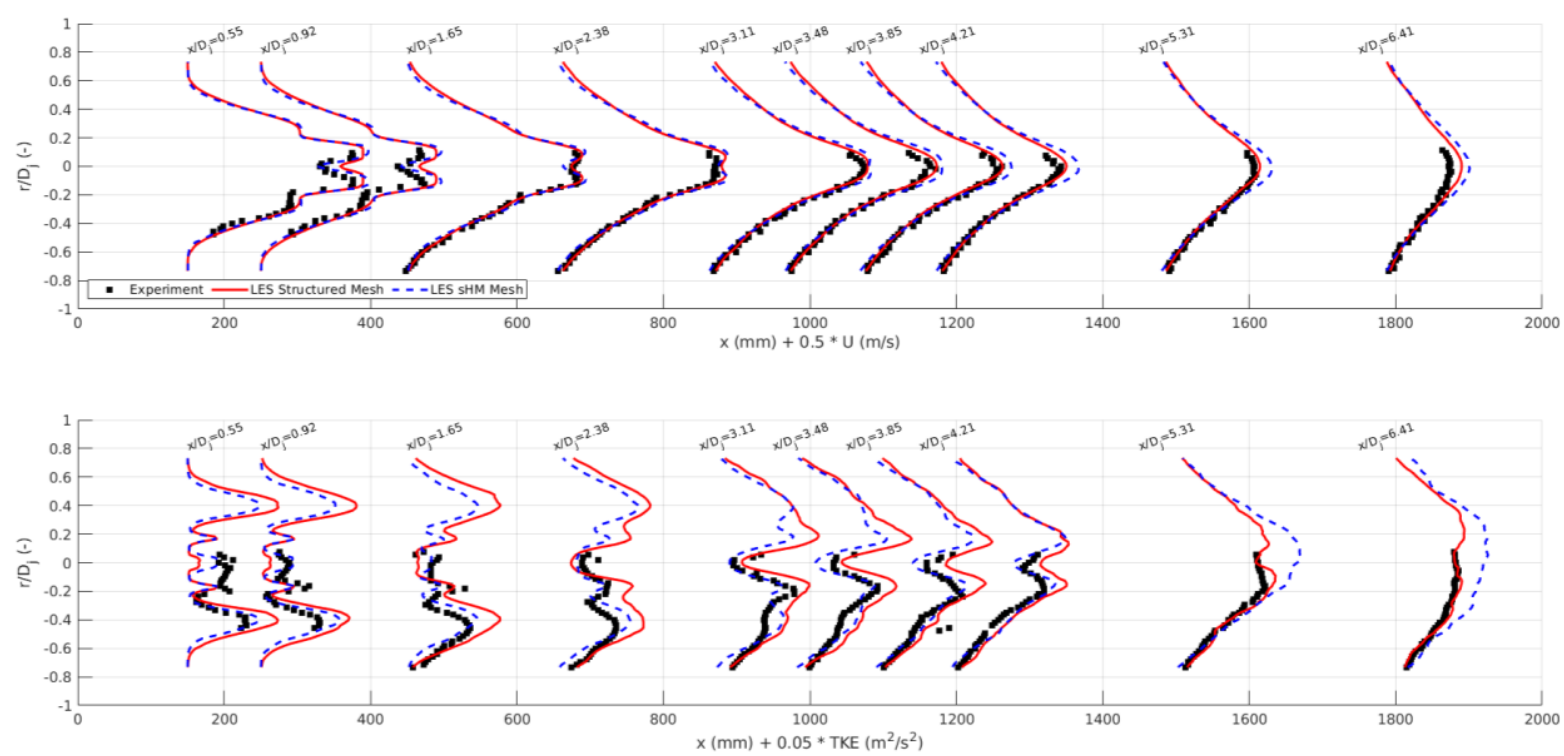

(b)

Fig. 8 Comparison of radial profiles of the mean axial velocity and the turbulent kinetic energy with experiment: (a) CABARET LES on the sHM grid, RANS/ILES on the structured multiblock grid and the axisymmetric RANS solution, (b) CABARET LES on the SHM grid and CABARET LES on the structured multiblock grid. The experimental data are digitized from [14].

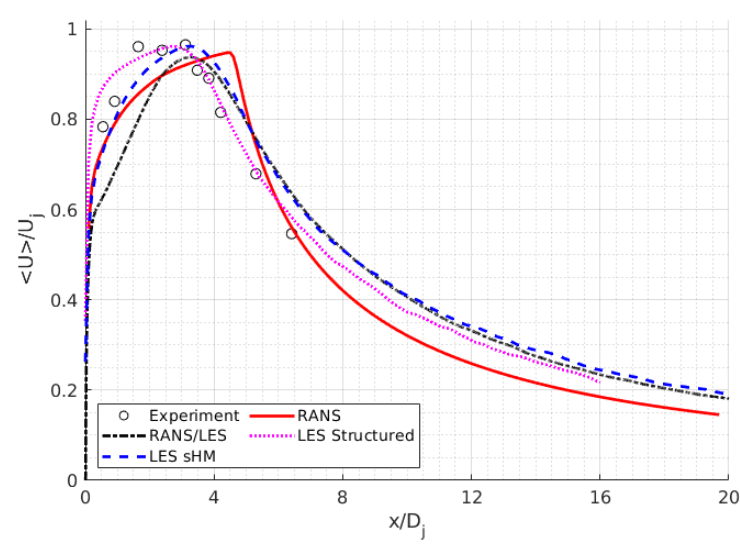

(a)

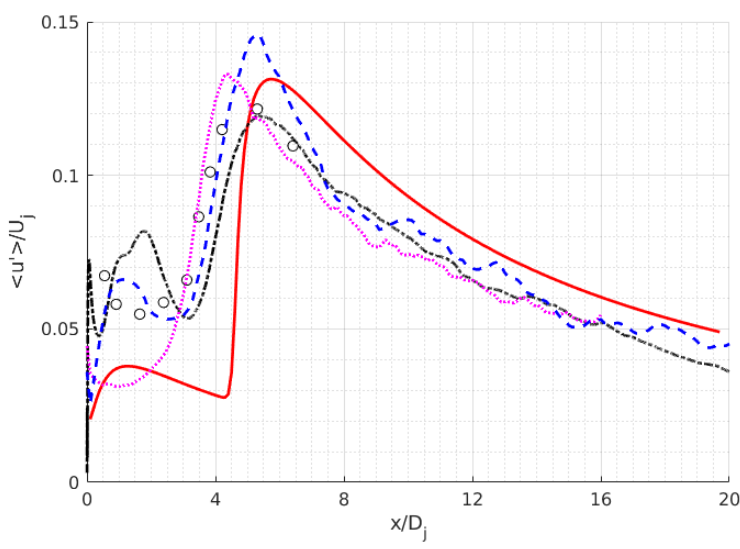

(b)

Fig. 9 Comparison of the CABARET solutions on the unstructured sHM and the structured multiblock grid, the RANS/ILES solution on the structured multiblock grid and RANS with the 
experiment: (a) centerline profile of the mean axial velocity and (b) centerline profile of the r.m.s. axial velocity fluctuations.

\section{B. Far-field acoustic modelling}

Fig.10 compares the noise spectra predictions of the CABARET method on the sHM grid and the RANS/ILES on the structured multiblock grid with the experiment for several polar angles from $30^{\circ}$ to $120^{\circ}$ to the jet flow. The Strouhal number definition is based on the bypass jet/nozzle parameters as discussed in Section IIIC. The vertical scale (line-to-line) in the plots is $10 \mathrm{~dB}$. The configurations of the acoustic control surfaces used for sounds predictions depend on the LES grid and differ one from another: the control surface for the sHM grid is closed with 16 discs while 3 closing discs with a larger space in-between are used for the structured grid. Furthermore, for the RANS-ILES method, two control surfaces are considered, which differ by the cone angle and the surface length (see Section IIIC).

The CABARET noise spectra predictions on the sHM grid are within $2 \mathrm{~dB}$ from the experiment for $0.06<\mathrm{St}_{\mathrm{D}}<10$ and most angles (some deterioration of accuracy to $2-3 \mathrm{~dB}$ is seen at the intermediate angles $60-70^{\circ}$ and frequencies $S t_{D}=1-2$ ). Most importantly, the peak noise spectra are captured within $1 \mathrm{~dB}$. The agreement with the experiment is particularly good at high frequencies, $\mathrm{St}_{\mathrm{D}}>2-3$.

The RANS/ILES noise spectra predictions on the two control surfaces are virtually the same. For frequencies, $0.2<\mathrm{St}_{\mathrm{D}}<8$ and polar angles from $40^{\circ}$ to $90^{\circ}$, the accuracy of the RANS-ILES noise predictions is within $2-3 \mathrm{~B}$ from the experiment. The predicted noise spectra show a drop off at frequencies $S t_{D}>8$. In the case of sound radiated in the downstream $\left(30^{\circ}\right)$ and upstream $\left(120^{\circ}\right)$ directions, the high-frequency spectra drop-off follows a 3-5dB "hump" in the noise spectra predictions in comparison with the experiment for frequencies $S t_{D}>2-3$. Furthermore, for frequencies lower than 0.2-0.4 and polar angles $90^{\circ}$ and higher, the RANS/ILES noise spectra predictions are 3-5 $\mathrm{dB}$ amplified in comparison with the experiment. The same accuracy is also obtained for the peak noise radiation angle $30^{\circ}$ polar angle, where the RANS-ILES solution underpredicts the noise peak level by $2-3 \mathrm{~dB}$. 
Fig. 11 shows the effect of the observed spectra difference on the overall sound power levels, where the frequency integration band is taken from Strouhal number 0.1 to 3.0 for all three datasets (the CABARET solution, the RANS/ILES solution, and the experiment) to produce the corresponding Band-Limited Over All Sound Pressure Levels (OASPL) directivity plot. The vertical scale of the figure is $5 \mathrm{~dB}$ (from line-to-line). The CABARET predictions are within $0.5-1 \mathrm{~dB}$ from the experiment for all polar angles except for $60-70^{\circ}$ angles which show $1-1.5 \mathrm{~dB}$ discrepancy with the experiment. Importantly, the peak sound levels are captured within $0.2 \mathrm{~dB}$.

The OASPL prediction of the RANS/ILES method are within 1-1.5 dB from the experiment for polar angles from $60^{\circ}$ to $100^{\circ}$. For downstream and upstream sound propagation angles, the discrepancy with the experiment (underprediction for small angles and overprediction for large angles) is $2-3 \mathrm{~dB}$.

The differences between the acoustic spectra predictions of the CABARET and RANS/ILES methods are striking given the good agreement between the flow solutions of the two methods (Fig.8a). In order to further analyse these differences, noise spectra predictions of the CABARET LES on the sHM grid and the structured multiblock grid using a similar acoustic integration surface with the same number of closing discs are compared in Fig.12 for three typical polar angles. In comparison with the noise predictions on the sHM grid, the noise spectra solutions on the structured grid result in a 3-5dB amplification in the high-frequency range $\mathrm{St}_{\mathrm{D}}>2-3$ for $30^{\circ}$ polar angle. This lift is similar to the "hump" observed in the RANS-ILES noise solution for the same frequencies and polar angle (Fig.10). Furthermore, the CABARET predictions on the structured grid show some 2-3dB underprediction of the noise peak at $30^{\circ}$ angle and also amplification of frequencies lower than $\mathrm{St}_{\mathrm{D}}=$ 0.05-0.1. Again, these are in consistence with the previously observed underprediction of the peak noise spectra of the RANS-ILES solution on the same grid. These revealed differences between the noise predictions of the CABARET method on the two grids can be attributed to the difference between the sHM grid and the structured multiblock in the initial jet flow region (see discussion in Section IIIB).

Finally, to analyse the effect of the number of closing discs and the distance between them, Fig.13 compares the spectra predictions of the CABARET LES on the structured multiblock grid with 16,3 , 
and 2 closing discs. The last two configurations are obtained by using discs 1,8 and 16 and also 1 and 16 out of the original 16 closing discs of the same acoustic surface (Fig.6). The 2-disc configuration is similar to the previously considered control surfaces of the RANS-LES method in Fig.11 (KS-0 and KS-1) in terms of the distance between the adjacent discs, while the 3-disc configuration has the same number of closing discs. The most prominent effect of the decrease of the number of closing discs is in the spurious rise of low frequency spectra which reaches $3-6 \mathrm{~dB}$ for $0.1<\mathrm{St}_{\mathrm{D}}<0.3$, which is general agreement with [12]. Besides, the effect of insufficient number of closing discs appears to be in the spectra drop off at high frequencies $\left(\mathrm{St}_{\mathrm{D}}>3-4\right)$, which partly balances the lift of high-frequency spectra on the structured grid for high observer angles, 60 and $90^{\circ}$ (compare with Fig.12). The last two effects of the reduced number of closing discs can explain the early drop off of the highfrequency noise spectra of the RANS-ILES solution and also the spurious lift of the low frequencies in comparison with the experiment and the CABARET solution (compare with Fig.10). It can be further argued that another factor, which helps improving the noise spectra predictions at highest frequencies $\left(\mathrm{St}_{\mathrm{D}}>8\right)$ is the synthetic turbulence inflow boundary condition as well as the lowdissipation property of the CABARET method.
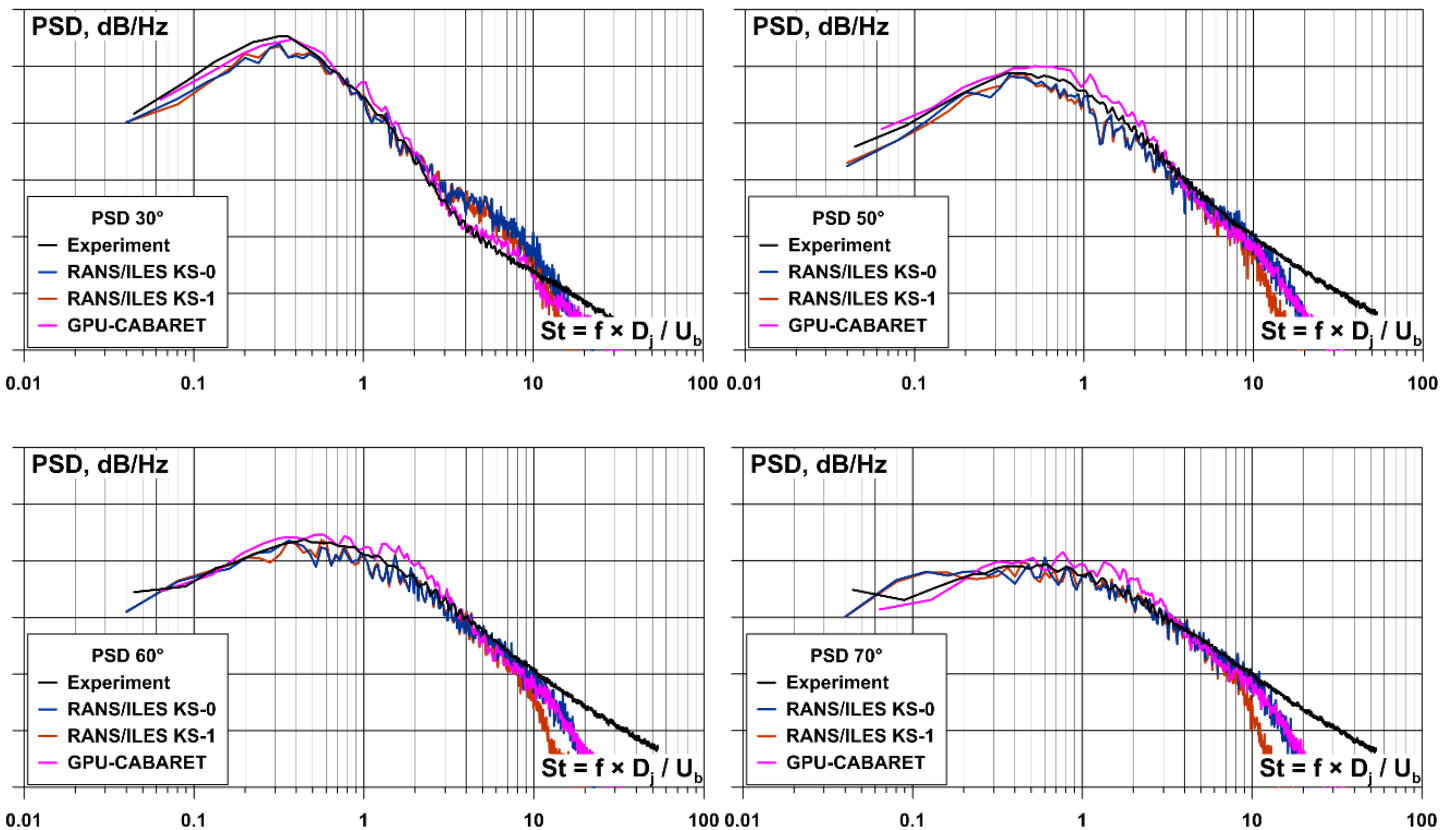

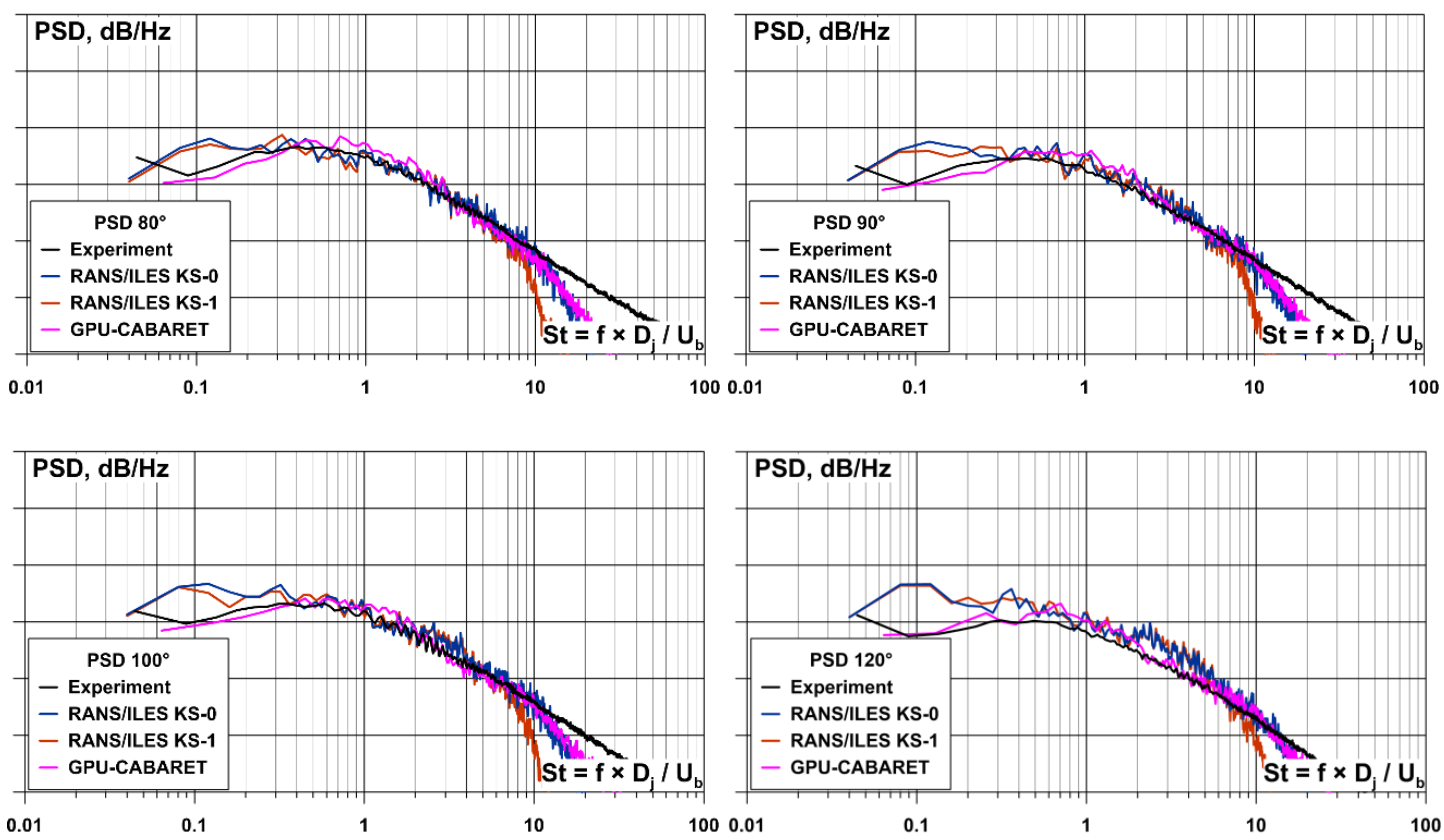

Fig. 10 Comparison of Power Spectra Density predictions with the experiment using the FW-H method based on the CABARET LES on the SHM grid and the RANS/ILES on the structured grid (KS-0 and KS-1 correspond to two different control surfaces on the structured grid). The dB scale in the plot is $10 \mathrm{~dB}$.

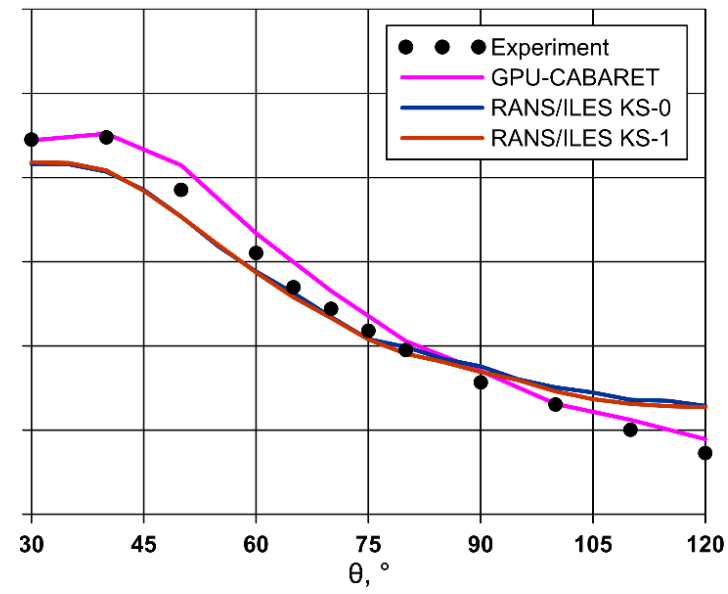

Fig. 11 Comparison of Band Limited Over All Sound Pressure Level predictions with the experiment using the FW-H method based on the CABARET LES on the SHM grid and the RANS/ILES on the structured grid (KS-0 and KS-1 correspond to two different control surfaces on the structured grid). The vertical scale is $5 \mathrm{~dB}$. 

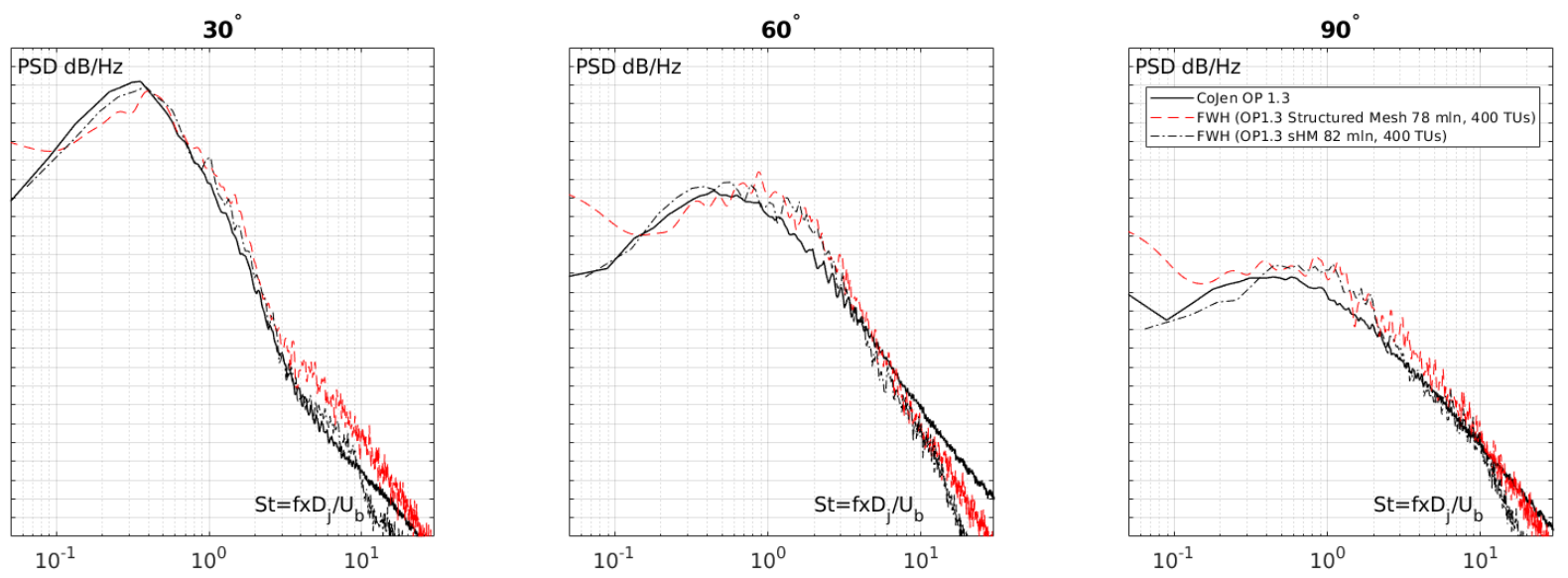

Fig. 12 Comparison of Power Spectra Density predictions with the experiment using the FW-H method based on the CABARET LES on the SHM grid and the structured grid based on 16 closing discs. The $\mathrm{dB}$ scale in the plot is $2 \mathrm{~dB}$.
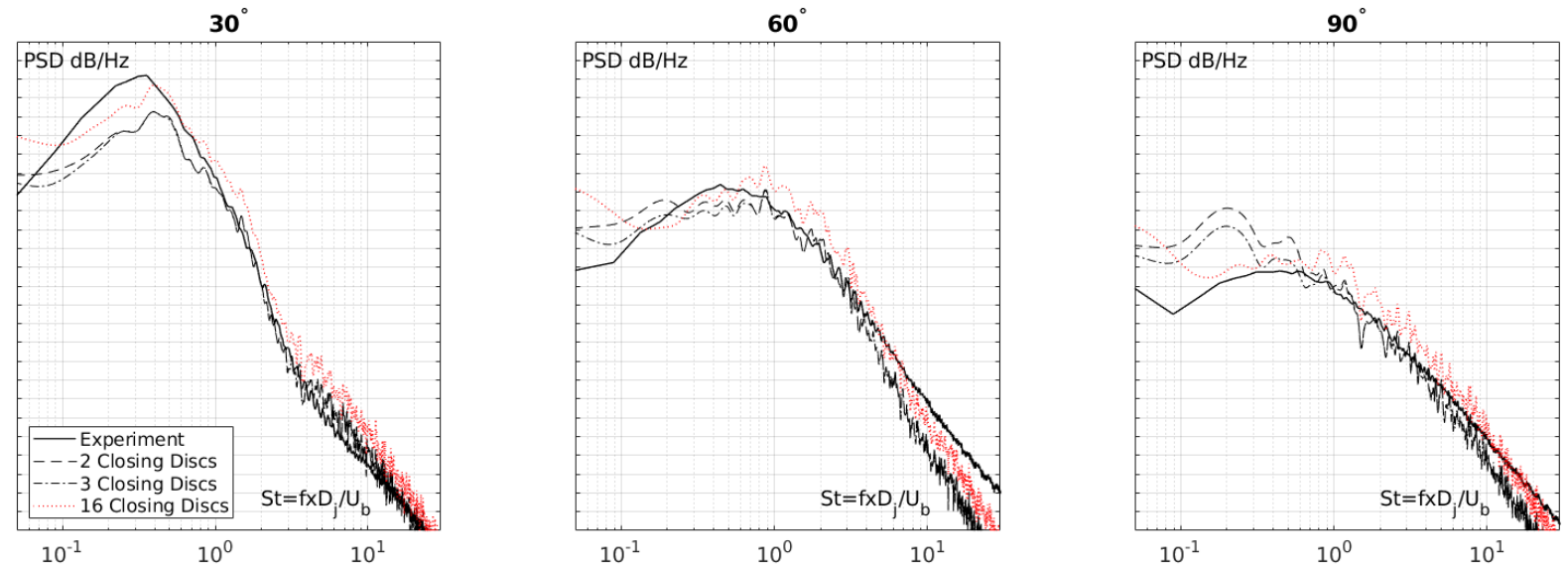

Fig. 13 Comparison of Power Spectra Density predictions with the experiment using the FW-H method based on the CABARET LES on the same structured grid based on different numbers of closing discs:16,3 and 2 . The $\mathrm{dB}$ scale in the plot is $2 \mathrm{~dB}$.

\section{Conclusion}

A consistent set of flow and noise data, which is representative of a high-speed hot-core jet issuing from a short-cowl co-axial nozzle with a bullet body and typical of commercial aero engines, is considered. The accuracy and computational speed of different approaches for flow and noise 
simulation using Large Eddy Simulation (LES) is compared. In each case, LES is coupled with the Ffowcs Williams - Hawkings method (FW-H). The test conditions correspond to the so-called Operation Point (OP) 1.3 jet case from the EU Computation of Coaxial Jet Noise (CoJeN) experiment and the baseline axisymmetric Short Cowl Nozzle (SCN) geometry.

The first LES approach is based on the high-resolution CABARET method for approximating the inviscid part of the governing Navier-Stokes equations on unstructured meshes. Three key properties of the current implementation of the CABARET LES method, which have been considered in some detail in the present publication, include asynchronous time stepping using a hierarchy of cell update groups tailored for the optimal CFL number for accuracy, a wall model, and a synthetic turbulent inflow boundary condition. The computer implementation of the current CABARET LES solver uses a small memory footprint which allows running it on Graphics Processing Units (GPUs) in the "compute under desk" mode using several "gaming" GPU cards or a few high-end GPUs. The GPUCABARET solutions with demonstrate a good scalability: reducing the overall LES grid size by a factor of 8 leads to the LES solution acceleration by a factor of 6.7-8.5 depending the size of the smallest grid cell. The second LES approach corresponds to a class of the state-of-the art hybrid RANS/Implicit LES method. The implemented hybrid approach is in the spirit of Improved Delayed Detached Eddy Simulation (IDDES). The same system of the Navier-Stokes equations and the turbulence model equation on the entire computational domain where the transition from RANS to LES is determined by a wall distance and flow parameters. Details of this procedure, which are somewhat different from the canonical DES approach are presented too. For solving the governing equations together with the turbulence model, a combination of a high-order Roe scheme and WENO is used together with implicit time stepping. The parallel implementation of the RANS/ILES method is run on a MPI cluster of 1600 computational cores. Two grid generation strategies for LES of coaxial jet flows are considered. One is based on using the OpenFOAM snappyHexMesh (sHM) utility which allows to place refined grid patches of good-quality Cartesian grid where they are needed near the nozzle geometry including the early shear layers. The generated instructed sHM grid of $82 \times 10^{6}$ cell grid has a good resolution in the initial and interaction region of the coaxial jet while its density in the mixed flow region downstream in the jet is less fine as a result of optimising the grid cell 
distribution. The second LES strategy considered corresponds to a conventional structured multiblock curvilinear body-fitted grid. It has $78 \times 10^{6}$ cells in total. In comparison with the sHM, the structured body-fitted grid is much smoother, has a $3.75 \mathrm{x}$ finer resolution in the wall normal direction, and also have a better resolution downstream in the mixed flow region for turbulence modelling. However, the structured grid generation also produces high-aspect-ratio anisotropic cells in the initial jet flow region especially near the complex geometry elements such as near the nozzle bullet. The run times of the GPU-CABARET method on two high-end GPU cards and the RANS-ILES method on 1600 computational cores on the same structured multiblock grid are the same within $10 \%$. In comparison with these, CABARET on the unstructured sHM grid is a factor of 1.67 faster.

In addition to the LES, Reynolds Averaged Navier Stokes (RANS) equations for the same nozzle geometry were obtained. The goal of the comparison with RANS was to evaluate trade-offs of using a faster but a lower-fidelity flow solution in comparison with LES for computing the meanflow velocity and turbulent kinetic energy in the co-axial jet flow, which quantities can be further used in low-order jet noise modelling schemes.

The agreement between the LES solutions and the experiment both the meanflow velocity, turbulent kinetic energy, and r.m.s fluctuation of stream-wise velocity components is good for jet locations $0.92 \leq x / D_{j} \leq 4.21$. This range of jet locations covers the second part of the initial region, the interaction region, and the beginning of the mixed flow region, which contain dominant noise sources of the coaxial jet. The LES solutions on the sHM grid tend to be more accurate in the initial jet flow region. The solutions on the structured multiblock grid are more accurate in the downstream part of the jet locations but less so in the initial jet region due to high-aspect-ratio anisotropic grid cells downstream of the bullet body. The importance of having good-quality isotropic grid cells in the initial jet flow region appears to be more dominant in comparison with the effect of the synthetic turbulent boundary condition. In comparison with LES, the RANS solution for turbulent kinetic energy shows discrepancies due to the underprediction of large-scale mixing in the jet core and also outside of jet shear layers due to limitations of the RANS turbulence model. Still, the RANS solution for the peak turbulent kinetic energy in the bypass/ambient shear layers, which is 
most important for low-order jet noise modelling [18], are in a reasonable agreement with LES for a range of jet locations.

For far-field noise modeling, the LES solutions are combined with the Ffowcs Williams Hawkings method. A retarded-time penetrable integration surface formulation with multiple closing discs is used following recommendations in the literature. Noise spectra predictions of the CABARET method on the sHM grid are within $2 \mathrm{~dB}$ from the experiment for $0.06<\mathrm{St}_{\mathrm{D}}<10$ where the Strouhal number is based on the bypass nozzle parameters for most polar angles in the region of $30^{\circ}$ to $120^{\circ}$ to the jet axis (apart from intermediate frequencies at angles $60-70^{\circ}$ where the accuracy is $2-3 \mathrm{~dB}$ ). Most notably, the peak of the noise spectra at $30^{\circ}$ angle is captured within $1 \mathrm{~dB}$. In comparison with this, the accuracy of noise spectra predictions of the RANS-ILES method on the structured multiblock grid shows some variation depending on the polar angle and frequency. For frequencies $0.2<\mathrm{St}_{\mathrm{D}}<8$ and polar angles $40-90^{\circ}$, the accuracy of the RANS-ILES noise spectra predictions is within 2-3B from the experiment. However, for noise radiated in the downstream $\left(30^{\circ}\right)$ and upstream $\left(120^{\circ}\right)$ directions, there is a marked $3-5 \mathrm{~dB}$ "hump" in the noise spectra predictions in comparison with the experiment for frequencies $\mathrm{St}_{\mathrm{D}}>2$ 2-3. Furthermore, for polar angles $90^{\circ}$ and higher, the RANS/ILES noise spectra predictions for frequencies $\mathrm{St}_{\mathrm{D}}<0.2-0.4$ show a 3-5 $\mathrm{dB}$ amplification in comparison with the experiment. The results of the two LES methods on the two different grids for Band Averaged OASPL directivity confirm the same trends as the revealed differences in noise spectra predictions.

To understand the differences between the noise predictions of the LES methods on different grids and with different acoustic surfaces of the FW-H method, noise spectra predictions of the CABARET LES on the structured multiblock grid are compared with the CABARET predictions on the sHM grid. In both cases, the configuration of the acoustic surfaces was very similar including the details of location and number of the closing discs of the acoustic integration surface. In a separate test, the FW$\mathrm{H}$ predictions based on the CABARET LES solution on the same structured grid for different numbers of closing discs are compared. The acoustic control surface with a reduced number of closing discs of the CABARET method mimics the acoustic surface configuration of the RANS-ILES method on the same grid. The comparison between the noise spectra produced from the CABARET LES solution on 
the two different grids suggests that the $3-5 \mathrm{~dB}$ high-frequency noise amplification at $30^{\circ}$ polar angle and 2-3dB peak noise underperdiction in the noise spectra solutions of the RANS-ILES method can be attributed to the anisotropy the structured multiblock grid in the initial jet flow region. The comparison of the noise predictions from different closing discs in the acoustic integration surface shows that an insufficient number of closing discs may lead to a faster attenuation of the highfrequency noise spectra as well as to a spurious 3-6dB lift of the low frequency spectra in comparison with the experiment.

\section{Acknowledgments}

The research has been supported by the Aero Acoustic Research Consortium administrated by Ohio Aerospace Institute, the Engineering and Physical Sciences Research Council (EP/S002065/1), and the Russian Science Foundation (Grant No. 19-12-00256).

The CoJeN data used in the current study have been produced by QinetiQ. The authors are grateful to Craig Mead for providing the CoJeN experiment noise data shown in Figs.10-13. Fruitful discussions with Drs Stewart Leib and Vasily Semiletov are gratefully acknowledged. 


\section{References}

[1] Fisher, M.J., and Preston, G.A., and Bryce, W.D., "A Modeling of the Noise from Simple Coaxial Jets,” AIAA-1993-4413, October 1993.

[2] Stone, J.R., Krejsa, E.A. and Clark, B.J., "Semi-Empirical Model for Coannular Nozzle Component Noise Extraction and Correlation Including the Effects of Noise Reduction Devices," AIAA-2003-1060, 2003.

[3] Khavaran, A. and Bridges, J. Jet Noise Scaling in Dual Stream Nozzles, NASA/TM-2010216887

[4] Tanna, H.K. 1977. An experimental study of jet noise: Part I turbulent mixing noise. J. Sound Vib., 50, 405-428.

[5] Bodony, D. and Lele, S. K. 2008. Current status of jet noise predictions using large-eddy simulation. AIAA J., 46:364-380.

[6] Bridges, J. and Wernet, M.P. 2011. The NASA Subsonic Jet Particle Image Velocimetry (PIV) Dataset, NASA/TM-2011-216807.

[7] Skeen, A. 2006 The development of high-speed PIV techniques and their application to jet noise measurement. PhD thesis, University of Warwick

[8] Tinney, C. E. and Jordan, P. 2008 The near pressure field of co-axial subsonic jets. J. Fluid Mech. 611, 175-204, DOI: 10.1017/S0022112008001833.

[9] Mead, C.J., Wrighton, C., Britchford, K. An Experimental Study of Co-Axial Jets Using Acoustic, PIV\&LDA Methods (CoJen), AIAA 2015-3122.

[10] Vuillemin, A., Loheac, P., Rahier, G., Vuillot, F. \& Lupoglazoff, N. 2005 Aeroacoustic numerical method assessment for a double stream nozzle. In 11th AIAA/CEAS Aeroacoustics Conference. DOI: 10.2514/6.2005-3043.

[11] Vuillot, F., Lupoglazoff, N., Rahier, G. Double-stream nozzles flow and noise computations and comparisons to experiments, 46th AIAA Aerospace Sciences Meeting and Exhibit 7 - 10 January 2008, Reno, Nevada.

[12] Shur, M. L., Spalart, P. R., Strelets, M. Kh. 2005. Noise Prediction for increasingly complex jets. Part I: Methods and tests. Part II: Applications Int. J. Aeroacoustics., 4(34), 21366. 
[13] Housman, J.A., Stich, G.-D., Kiris, C.C., Bridges, J. Jet Noise Prediction using Hybrid RANS/LES with Structured Overset Grids. 2017. 23rd AIAA/CEAS Aeroacoustics Conference, AIAA 2017-3213.

[14] Casalino, D. and Lele, S.K. Lattice-Boltzmann simulation of coaxial jet noise generation, Center for Turbulence Research, Proceedings of the Summer Program 2014.

[15] de Almeida, O., Barbosa, J.R.,Moro, J.B., Self, R.H. Noise Source Distribution of Coaxial Subsonic Jet-Short-Cowl Nozzle, J. Aerosp. Technol. Manag. Sao Jose dos Campos, vol 6, no1, pp 43-52, Jan-Mar, 2014

[16] Gloor, M.R. Numerical investigation on hydrodynamic stability and noise radiation of coaxial jet flows, PhD Thesis ETH Zurich, 2014

[17] Goldstein, M. E., "A generalized acoustic analogy," J. Fluid Mechanics, 488 (2003), pp. 315-333.

[18] Gryazev, V., Markesteijn, A.P. and Karabasov, S.A. 2019. Low-Order Models of Dual-Stream Jet Noise with Temperature Effects Based on the Goldstein Generalised Acoustic Analogy, 25th AIAA/CEAS Aeroacoustics Conference (Aeroacoustics 2019), 20 - 23 May 2019, Delft, The Netherlands, AIAA Paper 2019-2665.

[19] Ffowcs Williams, J. E. and Hawkings, D. L. Sound Generated by Turbulence and Surfaces in Unsteady Motion. Phil. Trans. Roy. Soc. Ser. A 264 (91141), 321 (1969).

[20] Markesteijn, A.P., Karabasov, S.A., Gryazev, V., Ayupov, R.S., Benderskiy, L. and Lyubimov, D. "Comparison of GPU CABARET, RANS and RANS/ILES High Resolution Method Solutions for the CoJeN Jet Noise Experiment”, 25th AIAA/CEAS Aeroacoustics Conference (Aeroacoustics 2019), 20 - 23 May 2019, Delft, The Netherlands, AIAA 2019-2446.

[21] Markesteijn, A.P. and Karabasov, S.A. GPU CABARET Solver Extension to Handle Complex Geometries Utilizing snappyHexMesh with Asynchronous Time Stepping, AIAA 2017-4184

[22] Markesteijn, A.P. and Karabasov, S.A. 2018. CABARET solutions on Graphics Processing Units for NASA jets: grid sensitivity and unsteady inflow condition effect, CR Mecanique (Proc. French Academy of Sciences), 346 (10), 948-963. 
[23] Markesteijn, A.P. and Karabasov, S.A. GPU CABARET Solutions for the CoJen Jet Noise Experiment, 2018 AIAA/CEAS Aeroacoustics Conference, AIAA AVIATION Forum, (AIAA 2018-3921)

[24] Goloviznin, V. M., Samarskii, A. A., Finite difference approximation of convective transport equation with space splitting time derivative, Jour Matem. Mod., 1998 , 10(1), 86-100.

[25] Karabasov, S. A., and Goloviznin, V. M., Compact Accurately Boundary Adjusting highREsolution Technique for Fluid Dynamics, J. Comput. Phys., 2009, 228, 7426-7451.

[26] Chintagunta, A., Naghibi, S.E., and Karabasov, S.A. Flux-corrected dispersion-improved CABARET schemes for linear and nonlinear wave propagation problems, Computers \& Fluids, August 2017.

[27] Goloviznin, V.M., Zaitsev, M.A., Karabasov, S.A., and Korotkin, I.A. 2013. New CFD Algorithms for Multiprocessor Computer Systems (in Russian), Mosk. Gos. Univ, Moscow, ISBN: 978-5-211-06426-3

[28] Semiletov, V. A., and Karabasov, S. A., CABARET scheme with conservation-flux asynchronous time-stepping for nonlinear aeroacoustics problems, J. Comp.Physics, 2013, 253(15), 157165.

[29] Semiletov, V. A. and Karabasov, S. A. "CABARET scheme for computational aero acoustics: extension to asynchronous time stepping and 3D flow modelling”, International Journal of Aeroacoustics 13 (3-4), 321-336.

[30] Faranosov, G. A., Goloviznin, V. M., Karabasov, S. A., Kondakov, V. G., Kopiev,V. F., Zaitsev, M. A. 2013. CABARET method on unstructured hexahedral grids for jet noise computation. Comp. and Fluids, 88, 165-179.

[31] Bodart, J. and Larsson, J. 2011. "Wall-modeled large eddy simulation in complex geometries with application to high-lift devices.” Annual Research Briefs, Center for Turbulence Research, Stanford University, pp. 37-48.

[32] Park, G. I. and Moin, P. 2016. "Numerical aspects and implementation of a two-layer zonal wall model for LES of compressible turbulent flows on unstructured meshes.” J. Comput. Phys. 305, 589-603. 
[33] Mukha, T., Rezaeiravesh, S. and Liefvendahl, M. 2018. "A Library for Wall-Modelled LargeEddy Simulation Based on OpenFOAM Technology.”

[34] Saad, T. Cline, D., Stoll, R. and Sutherland, J.C. 2014. Scalable Tools for Generating Synthetic Isotropic Turbulence with Arbitrary Spectra. http://dx.doi.org/10.2514/1.J055230.

[35] Richards, A., Saad, T. and Sutherland, J.C. 2018. A Fast Turbulence Generator using Graphics Processing Units", AIAA AVIATION Forum, (AIAA 2018-3559). https://doi.org/10.2514/6.2018-3559

[36] Lyubimov, D.A., Chestnykh A.O. Flow in a High-Velocity Mixed Compression Inlet Studied by the RANS/ILES Method in Different Operation Modes. High Temperature, 2018, Vol. 56, No. 5, pp.702-710.

[37] Lyubimov D. A. Development and Application of a High_Resolution Technique for Jet Flow Computation Using Large Eddy Simulation. High Temperature, 2012, V. 50, No. 3, pp. 420-436.

[38] Suresh A., Huynh H. T. Accurate Monotonicity_Preserving Schemes with Runge-Kutta Time Stepping. J. Comp. Physics. 1997. V.136. No 1. pp.83-99.

[39] Spalart P.R., Allmaras S.R.A One-Equation Turbulence Model for Aerodynamic Flows. La Recherche Aerospatiale. 1994. No 1. pp. 5-21.

[40] T. Unfer, J.-P. Boeuf, F. Rogier, F. Thivet, An Asynchronous Scheme with Local Time Stepping for Multi-scale Transport Problems: Application to Gas Discharge, Journal of Computational Physics 227, 898-918, 2007

[41] J.C. Yen, Demonstration of a Multi-Dimansional Time-Accurate Local Time Stepping CESE Method, 17th AIAA/CEAS Aeroacoustics Conference (32nd AIAA Aeroacoustics Conference), Portland, Oregon, 5-8 June 2011

[42] Bailly, C. andJuve, D.. A stochastic approach to compute subsonic noise using linearized Euler's equations, In 5th AIAA/CEAS Aeroacoustics Conference and Exhibit, 1999, AIAA 991872.

[43] Karabasov, S.A., Bogey, C., and Hynes, T.P. 2013. An investigation of the mechanisms of sound generation in initially laminar, subsonic jets using the Goldstein acoustic analogy, J. Fluid Mech. Vol. 714, $24-57$. 
[44] Spalart P.R., Jou W. H., Strelets M., Allmaras S.R. Comments on the feasibility of LES for wings, and on a hybrid RANS/LES approach. First AFOSR International conference on DNS/LES. Rouston, Louisiana, USA, 1997, pp.669-687.

[45] Shur M.L., Spalart P.R., Strelets M.K., Travin A.K. A Hybrid RANS-LES Approach with Delayed-DES and Wall-modeled LES Capabilities. Int. J. Heat Fluid Flow. 2008. V. 29. P. 1638.

[46] Semiletov VA and Karabasov SA. 2018. A volume integral implementation of the Goldstein generalised acoustic analogy for unsteady flow simulations. J. Fluid Mech. 853, 461-487.

[47] Bendersky L.A., Lyubimov D.A., Potekhina I.V., Fedorenko A.E. Application of the highresolution RANS/ILES technology for flow simulation and the nearby acoustics field of nearwall jets and mixing layers. TsAGI Science Journal. 2016. Vol. 47. No. 2. pp. 159-183. https://doi.org/10.1615/TsAGISciJ.2016017766

[48] Ayupov R.Sh., Benderskii L.A., Lyubimov D.A. RANS/ILES Analysis of the Flow Pattern and the Acoustic Characteristics of a Supersonic Off-Design Jet at Large Nozzle Pressure Ratios. Fluid Dynamics. 2019. Vol. 54. pp. 114-122. https://doi.org/10.1134/S0015462819010038 